\title{
Numerical Model of Sedimentation/Thickening WITH INERTIAL EFFECTS
}

\author{
By Joanna R. Karl ${ }^{1}$ and Scott A. Wells, ${ }^{2}$ Member, ASCE
}

\begin{abstract}
Aвstract: A numerical model of gravity sedimentation and thickening was developed from the governing two-phase flow equations for the liquid and solid phases. The inertial and gravity terms in the solid and liquid momentum equations were retained in the gravity sedimentation and thickening model. An implicit, spacestaggered finite-difference algorithm was developed for the resulting coupled partial differential equations. Constitutive relationships describing the physical properties of the slurry were required to solve the numerical model. These constitutive properties describing the relationship between effective stress and porosity and between permeability and porosity were determined experimentally and by model calibration. The model was calibrated and verified using the data of dynamic porosity profiles of gravity sedimentation and thickening of kaolin suspensions in distilled water.
\end{abstract}

\section{INTRODUCTION}

A large fraction of the current cost of wastewater treatment is from the treatment and disposal of sludge (Evans and Filman 1988). Improved design and performance of sedimentation and thickening facilities could decrease treatment and disposal costs. Thickening and dewatering facilities are designed based on field experience, trial and error, and pilot plant testing. Refinement of computer simulation models of sedimentation/thickening processes based on slurry properties would enable engineers to optimize the process design.

A numerical model of the physics of gravity sedimentation and thickening was developed, calibrated, and verified using porosity data from the sedimentation and thickening of kaolin clay suspensions. Dynamic porosity profiles of gravity sedimentation and thickening of kaolin clay suspensions in distilled water were obtained using an X-ray attenuation technique at the Cornell High Energy Synchrotron Source (CHESS). Experimental details of this procedure are shown in Wells (1990) and Bierck et al. (1988).

\section{GRAVITY SEDIMENTATION AND THICKENING MODELING}

\section{Sedimentation}

In general, gravity sedimentation is considered to occur as four different types, often with two or more phenomena occurring simultaneously:

- Discreet particle settling (low solids concentration with no significant interaction between particles, i.e., no flocculation)

- Flocculent settling (dilute suspensions that coalesce, thus increasing their mass and settling faster)

- Hindered settling (suspensions where the concentration is great enough to develop particle contact as they settle, which causes their flow pattern to be modified, and may or may not be flocculating)

- Compression (from the continuously increasing particle weight)

\footnotetext{
${ }^{1}$ Sr. Engr., Regional Envir. Mgmt., Metro, Portland, OR 97232-2736. 0751.

'Prof., Dept. of Civ. Engrg., Portland State Univ., Portland, OR $97207-$

Note. Associate Editor: Lewis A. Rossman. Discussion open until February 1,2000 . To extend the closing date one month, a written request must be filed with the ASCE Manager of Journals. The manuscript for this paper was submitted for review and possible publication on July 20 , 1998. This paper is part of the Journal of Environmental Engineering, Vol. 125, No. 9, September, 1999. C)ASCE, ISSN 0733-9372/99/00090792-0806/\$8.00 + \$.50 per page. Paper No. 18821.
}

The gravity sedimentation process will proceed differently, based on whether the suspension is flocculating or nonflocculating. Materials such as wastewater sludges tend to flocculate as they thicken, as compared with coarse mineral particulates that do not (Kos 1985), and the flocs hold water that can only be expelled from the sediment by compression (Concha and Bustos 1985).

Gravity sedimentation research prior to 1950 was mostly based on Stokes' law, formulated in 1851, which described the settling of discreet particles in a low solids concentration. Stokes assumed the settlement to occur as a steady-state process. Coe and Clevenger (1916) noted a difference in settling behavior between suspensions with either coarser or finer particles. In the case of the coarser particles, both sedimentation and settling proceeded at a constant rate, whereas in very fine flocculated slimes, they noted a progressively decreasing rate of sedimentation and a varying settlement rate (Richardson and Zaki 1954; Schiffman et al. 1985). The theory of hindered settling was observed by Kynch (1952) and showed that the settling process during this mode is very transient and that continuity equations are required to model its physics. He further suggested there was only one solids velocity for a given concentration, which led to the concept of developing slurryspecific flux curves (Kos 1985).

A number of researchers (Michaels and Bolger 1962; Fitch 1966, 1975, 1979, 1983; Shirato et al. 1970; Tiller 1981) noted that Kynch's theory is not valid in the compression zone of flocculated suspensions, where the particle velocity is dependent on the solids stress gradient as well as the concentration (Fitch 1983). Studies by Been (1980), Tiller (1981), and Fitch (1983) have generalized Kynch's theory to include the consolidation process.

\section{Gravity Thickening Models}

Gravity thickening is the application of gravity sedimentation as a separation process of solid particles and liquid. The gravity thickening models utilize a force balance (or momentum) equation. The differences between the various models are the terms that are considered and the constitutive relationship assumptions utilized (Fitch 1979; Vaccari and Uchrin 1989).

In the Michaels and Bolger (1962) batch thickening model, the settling velocity is a function of concentration and concentration gradient (Vaccari and Uchrin 1989). Fitch (1966) further developed the Michaels and Bolger model to apply to continuous thickening as well. Kos (1977) developed two more models, which accounted for the sensitively varying permeability in the solids stress gradient (Vaccari and Uchrin 1989). Calibrating these models required rigorous measurements of porosity and particle stress (Vaccari and Uchrin 1989). Based on the Kos models, Vaccari and Uchrin (1989) 
developed a new model with solid velocity as a function only of the concentration and concentration gradient, thus resulting in much simpler calibration techniques.

Although the inertial effects have been ignored in the force balance equation by most researchers, Dixon et al. (1976) determined that inertial effects in sedimentation could not always be neglected. Dixon (1979) studied batch thickening of an initially uniform suspension, and concluded that inertia may be important in the free settling zone. According to Wakeman and Holdich (1984), the inertial effects were small throughout a settling column. Dixon et al. (1985) emphasized that the region where inertia was important was the narrow thickening region interface between suspension and sediment where rapid velocity change was occurring.

Hence, the gravity sedimentation model developed for this project has the following characteristics:

- Variable, nonlinear constitutive relationships for permeability and effective stress (as functions of porosity)

- Inclusion of hindered settling based on the constitutive relationship for permeability as a function of porosity with effective stress set to zero

- Inclusion of inertial terms in the solid momentum equation to evaluate their relative importance

The derivation of this model is described below.

\section{TWO-PHASE FLOW GOVERNING EQUATIONS}

\section{Summary of Initial Two-Phase Flow Governing Equations}

The gravity sedimentation and thickening model was based on four governing equations (Willis 1983; Soo 1989; Wells 1990; Karl 1993): liquid and solid continuity [(1) and (2)] and liquid and solid momentum [(3) and (4)].

Liquid continuity:

$$
\frac{\partial \varepsilon}{\partial t}=-\frac{\partial}{\partial z}\left(\varepsilon V_{l}\right)
$$

Solid continuity:

$$
\frac{\partial \varepsilon}{\partial t}=\frac{\partial}{\partial z}\left((1-\varepsilon) V_{s}\right)
$$

Liquid momentum:

$$
\begin{aligned}
& \varepsilon \rho_{l} \frac{\partial V_{l}}{\partial t}+\varepsilon \rho_{l} V_{l} \frac{\partial V_{l}}{\partial z}=-\varepsilon \rho_{l} g-\varepsilon F\left(V_{l}-V_{s}\right)-\varepsilon \frac{\partial p}{\partial z}
\end{aligned}
$$

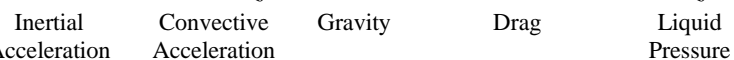

Solid momentum:

$$
\begin{array}{ccc}
(1-\varepsilon) \rho_{s} \frac{\partial V_{s}}{\partial t}+(1-\varepsilon) \rho_{s} V_{s} \frac{\partial V_{s}}{\partial z} & -(1-\varepsilon) \rho_{s} g \\
\begin{array}{c}
\text { Inertial } \\
\text { Acceleration } \\
\text { Convective } \\
\text { Acceleration }
\end{array} & \text { Gravity } \\
\text { Drag } & \begin{array}{c}
\text { Liquid } \\
\text { Pressure }
\end{array} & \begin{array}{c}
\text { Intergranular } \\
\text { Stresses }
\end{array}
\end{array}
$$

in which $\varepsilon=$ porosity (volume liquid/total volume); $t=$ time $(\mathrm{T}) ; z=$ distance from filtration medium $(\mathrm{L}) ; V_{l}=$ true liquid velocity (in contrast to Darcy velocity, $\left.\varepsilon V_{l}\right)(\mathrm{L} / \mathrm{T}) ; V_{s}=$ velocity of the solid particles $(\mathrm{L} / \mathrm{T}) ; F=\varepsilon \mu / k=$ averaged interfacial interaction term between the solid and the liquid phases (M/ $\left.\mathrm{L}^{3}-\mathrm{T}\right) ; \mu=$ dynamic (or absolute) viscosity (M/L-T); $k=$ intrinsic permeability $\left(\mathrm{L}^{2}\right) ; p=$ fluid static pressure $\left(\mathrm{M} / \mathrm{L}-\mathrm{T}^{2}\right) ; g$ $=$ acceleration due to gravity $\left(\mathrm{L} / \mathrm{T}^{2}\right) ; \rho_{l}=$ liquid density $(\mathrm{M} /$
$\left.\mathrm{L}^{3}\right) ; \rho_{s}=$ solid density $\left(\mathrm{M} / \mathrm{L}^{3}\right) ;$ and $\sigma^{\prime}=$ effective stress $\left(\mathrm{M} / \mathrm{L}-\mathrm{T}^{2}\right)$.

According to Dixon et al. (1985), the inertial terms are important in the interface between suspension and sediment, where rapid velocity change is occurring. In this narrow interface the particles, which have been settling at the terminal velocity corresponding to the initial concentration, are retarded (the velocity is near zero in the sediment) as they strike the top of the sediment. Thus, the inertial terms were retained in the gravity sedimentation and thickening model and were evaluated later as to their relative importance.

\section{Constitutive Relationships}

Two constitutive properties were required to close the set of governing equations: (1) $k$ as a function of $\varepsilon$, $t$, etc.; and (2) $\sigma^{\prime}$ as a function of $\varepsilon$, $t$, etc. These relationships reflect the properties of the slurry. In this development, permeability was assumed to be a function only of the local porosity, and the effective stress relationship was defined by the volume compressibility coefficient (Das 1983; Wells and Dick 1993)

$$
m_{v}=-\frac{\partial \varepsilon}{\partial \sigma^{\prime}}
$$

and $m_{v}$ is thus only a function of $\varepsilon$.

\section{Final Form of Governing Equations in Model Formulation}

The solid and liquid continuity equations can be equated as follows:

$$
\frac{\partial \varepsilon}{\partial t}=-\frac{\partial\left(\varepsilon V_{l}\right)}{\partial z}=\frac{\partial\left[(1-\varepsilon) V_{s}\right]}{\partial z}
$$

Integrating (6) from $z=0$ (at the bottom of the sedimentation/thickening vessel), where $\varepsilon V_{l}=\varepsilon_{0} V_{0}$ and $V_{s}=0$, to $z$

$$
-\int_{\varepsilon_{0} V_{0}}^{\varepsilon V_{l}} \partial\left(\varepsilon V_{l}\right)=\int_{0}^{V_{s}} \partial\left[(1-\varepsilon) V_{s}\right]
$$

Simplifying, (7) becomes

$$
V_{l}=\frac{\varepsilon_{0} V_{0}-(1-\varepsilon) V_{s}}{\varepsilon}
$$

where $\varepsilon_{0}=$ porosity at $z=0 ; V_{0}=$ true liquid velocity at $z=$ $0(\mathrm{~L} / \mathrm{T})$; and $\varepsilon_{0} V_{0}$ represents the liquid velocity leaving the sedimentation/thickening vessel.

Similarly, the two momentum equations can be added, resulting in a total momentum equation

$$
\begin{aligned}
\rho_{l} \varepsilon & \frac{\partial V_{l}}{\partial t}+\rho_{l} \varepsilon V_{l} \frac{\partial V_{l}}{\partial z}+(1-\varepsilon) \rho_{s} \frac{\partial V_{s}}{\partial t}+(1-\varepsilon) \rho_{s} V_{s} \frac{\partial V_{s}}{\partial z} \\
& =-\frac{\partial P}{\partial z}-\left[(1-\varepsilon) \rho_{s}+\varepsilon \rho_{l}\right] g-\frac{\partial \sigma^{\prime}}{\partial z}
\end{aligned}
$$

The following technique for simplifying these governing equations is similar to a technique described by Soo (1989). By equating $\partial P / \partial z$ in the solid momentum [(4)] and the total momentum [(9)], substituting $V_{l}$ from the total continuity [(8)] into (9), combining like terms, and substituting the constitutive relationship $m_{v}=-\partial \varepsilon / \partial \sigma^{\prime}$, (9) becomes

$$
\begin{aligned}
& {\left[(1-\varepsilon) \rho_{l}+\varepsilon \rho_{s}\right] \frac{\partial V_{s}}{\partial t}} \\
& \quad+\left[\rho_{l}\left(\varepsilon_{0} V_{0}-(1-\varepsilon) V_{s}\right)\left(\frac{1-\varepsilon}{\varepsilon}\right)+\varepsilon \rho_{s} V_{s}\right] \frac{\partial V_{s}}{\partial z}
\end{aligned}
$$




$$
\begin{aligned}
& +\left[-\rho_{l} \frac{\left(V_{s}-\varepsilon_{0} V_{0}\right)}{\varepsilon}\right] \frac{\partial \varepsilon}{\partial t}+\left[\frac{\rho_{l}}{\varepsilon^{2}}\left(\varepsilon_{0} V_{0}-V_{s}\right)\left(\varepsilon_{0} V_{0}-(1-\varepsilon) V_{s}\right)\right] \frac{\partial \varepsilon}{\partial z} \\
& +\left[-\rho_{l} \frac{\partial}{\partial t}\left(\varepsilon_{0} V_{0}\right)\right]=\varepsilon g\left(\rho_{l}-\rho_{s}\right)+\left(\frac{F}{1-\varepsilon}\right) \underset{\left(\varepsilon_{0} V_{0}-V_{s}\right)}{7} \\
& +\frac{\varepsilon}{(1-\varepsilon) m_{v}} \frac{\partial \varepsilon}{\partial z}
\end{aligned}
$$

Details of the derivation of (10) are shown in Appendix I.

Once the constitutive parameters $\left(F\right.$ and $\left.m_{v}\right)$ were known, (2) and (10) provided two equations with two unknowns: $V_{s}$ and $\varepsilon$.

\section{NUMERICAL SOLUTION TECHNIQUE}

The final governing equations were put into finite-difference form and solved numerically using appropriate boundary conditions. Eq. (2) was first solved to determine $\varepsilon$ at the new time level $n+1$. Using this result, (10) was used to solve for $V_{s}$ at the new time level $n+1$.

This model was formulated to account for sedimentation/ thickening with and without drainage by changing its boundary conditions (Karl 1993). Model simulation predictions were compared with gravity sedimentation/thickening data of kaolin suspensions determined at CHESS by Wells (1990) without drainage.

A "space-staggered mesh" was employed such that porosity $\varepsilon$ was evaluated at the control volume center, and solid velocity $V_{s}$ was evaluated at the control volume edges, as shown in Fig. 1.

\section{Boundary Conditions for $\varepsilon$ and $V_{s}$}

Boundary conditions were determined as follows for the case of gravity sedimentation/thickening without drainage, where $V_{0}=0$.

Top $(z=L)$

$$
\begin{gathered}
\text { Solid velocity: } \frac{\partial V_{s}}{\partial z}=0 \text { or }\left(V_{s}\right)_{k+2}=\left(V_{s}\right)_{k} \\
\text { Porosity: } \varepsilon_{k+1 / 2}=1.0
\end{gathered}
$$

where $k$ refers to the grid point number (Fig. 1).

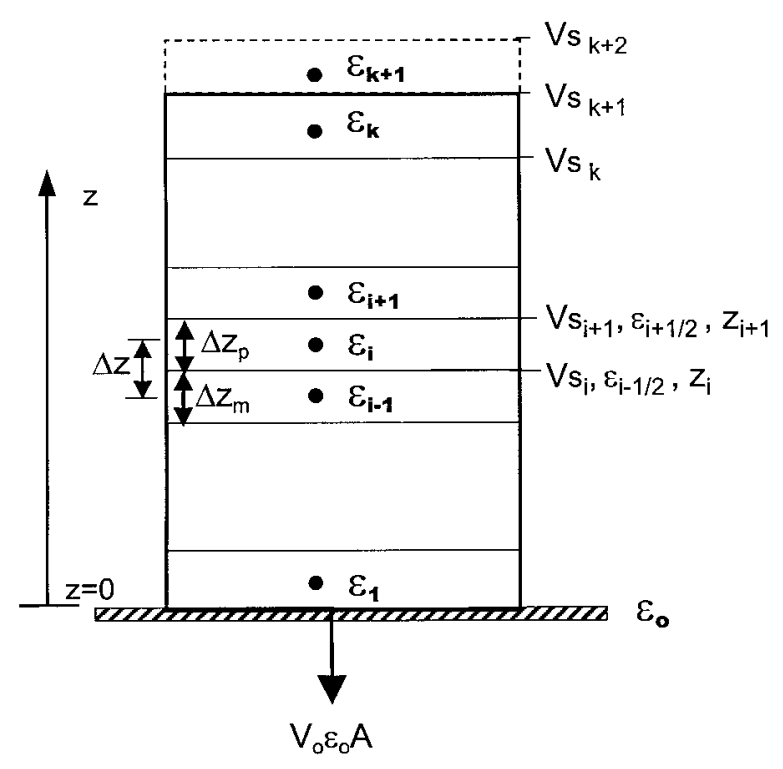

FIG. 1. Model Grid Layout
Bottom $(z=0)$

$$
\text { Solid velocity: } V_{s}=0 \text { or }\left(V_{s}\right)_{i=1}=0
$$

Porosity: The initial porosity at the bottom of the domain was calculated knowing the initial suspended solids concentration $C_{i}$ and solids density $\rho_{s}$ [i.e., $\varepsilon=1-C_{i} / \rho_{s}$. Porosities at the bottom of the domain for each new time level $n+1$ were then calculated using (2).

These boundary conditions reflect no flux of solids into the top or out from the bottom of the domain.

\section{Finite-Difference Form of (2)}

An explicit formulation was used to solve (2), which had a forward difference in time and central difference in space

$$
\frac{\varepsilon_{i}^{n+1}-\varepsilon_{i}^{n}}{\Delta t}=\frac{\left[(1-\varepsilon) V_{s}\right]_{i+1 / 2}^{n}-\left[(1-\varepsilon) V_{s}\right]_{i-1 / 2}^{n}}{\Delta z}
$$

Because this numerical scheme is unconditionally unstable for convection-dominated systems, artificial numerical diffusion was added when the system became convection-dominated. This occurred when a suspension had a very low initial solids concentration. In nonconvection dominated systems, artificial diffusion was not required. Adams and Rodi (1990) similarly used a hybrid numerical scheme based on whether the system was convection- or diffusion-dominated.

In contrast to an upwind scheme for (14), the centered space scheme provided increased numerical accuracy as long as the system was not convection-dominated. For the model user to control how much numerical diffusion was added to the solution, the artificial viscosity concept was introduced, as discussed later.

\section{Finite-Difference Form of (10)}

Eq. (10) was put into a general explicit-implicit finite-difference scheme, as shown in Table 1. To do so, the first step was to simplify the equation by renaming the coefficients (Table 1), as follows:

$$
\begin{aligned}
& A \frac{\partial V_{s}}{\partial t}+\left(B_{1}+B_{2} V_{s}\right) \frac{\partial V_{s}}{\partial z}+\left(C V_{s}+D\right) \\
& \begin{array}{lll}
1 & 2 & 3
\end{array} \\
& +\left(E+\left(F+G_{1}\right) V_{s}+G_{2} V_{s}^{2}\right)+H=I+\left(J+K V_{s}\right)+L
\end{aligned}
$$

Table 1 shows (15) with the $V_{s}$ term prior to discretization, the discretized or finite-difference form of the $V_{s}$ term (as either a function of time or in both its explicit and implicit formulation), and the coefficient of the term. This table is discussed further, below, when comparing the explicit and implicit solution strategies, comparing the effect of using a central difference versus an upwind difference of the spatial derivative of the solid velocity, and describing the linearization of the nonlinear terms.

\section{Explicit versus Implicit Strategy for “Momentum', Equation}

The fully explicit formulation for the momentum equation required a very small $\Delta t$ to remain stable, resulting in a lengthy computational time. The implicit solution technique allowed for larger time steps, while avoiding excessive buildup of round-off error (Farlow 1982).

To use the implicit scheme, a system of simultaneous linear algebraic equations was written for the linearized differential equation, and a Gaussian elimination procedure was used to solve these equations. The resulting coefficient matrix can be 
TABLE 1. Discretization of Eq. (15)

\begin{tabular}{|c|c|c|c|c|}
\hline \multirow[b]{2}{*}{$\begin{array}{c}\text { Term No. } \\
\text { (1) } \\
\end{array}$} & \multirow[b]{2}{*}{$\begin{array}{l}V_{s} \text { term: } \\
(2) \\
\end{array}$} & \multicolumn{2}{|c|}{ Discretization of $V_{s}$ term: } & \multirow[b]{2}{*}{$\begin{array}{c}\text { Coefficient } \\
\text { (at timestep } n \text { ) } \\
(5) \\
\end{array}$} \\
\hline & & $\begin{array}{l}\text { Explicit term: } \\
\left(V_{s} \text { term }\right)^{n} \\
\text { (3) }\end{array}$ & $\begin{array}{l}\text { Fully implicit term: } \\
\left(V_{s} \text { term }\right)^{n+1} \\
(4)\end{array}$ & \\
\hline 1 & $\frac{\partial V_{s}}{\partial t}$ & & $\frac{\left(V_{s}\right)_{i}^{n+1}-\left(V_{s}\right)_{i}^{n}}{\Delta t}$ & $A=(1-\varepsilon) \rho_{1}+\varepsilon \rho_{s}$ \\
\hline \multirow[t]{2}{*}{2} & $\frac{\partial V_{s}}{\partial z}$ & $\begin{array}{l}\text { Central difference: } \\
\frac{\left(V_{s}\right)_{i+1}^{n}-\left(V_{s}\right)_{i-1}^{n}}{2 \Delta z} \\
\text { Upwind: } \\
\frac{\left(V_{s}\right)_{i+1}^{n}-\left(V_{s}\right)_{i}^{n}}{\Delta z}\end{array}$ & $\begin{array}{l}\text { Central difference: } \\
\frac{\left(V_{s}\right)_{i+1}^{n+1}-\left(V_{s}\right)_{i-1}^{n+1}}{2 \Delta z} \\
\text { Upwind: } \\
\frac{\left(V_{s}\right)_{i+1}^{n+1}-\left(V_{s}\right)_{i}^{n+1}}{\Delta z}\end{array}$ & $B_{1}=\left(\frac{(1-\varepsilon)}{\varepsilon}\right) \rho_{1} \varepsilon_{0} V_{0}$ \\
\hline & $\begin{array}{l}V_{s} \frac{\partial V_{s}}{\partial z} \\
=\frac{\partial\left(\frac{V_{s}^{2}}{2}\right)}{\partial z}\end{array}$ & $\begin{array}{l}\text { Central difference: } \\
\frac{\left(\frac{V_{s}^{2}}{2}\right)_{i+1}^{n}-\left(\frac{V_{s}^{2}}{2}\right)_{i-1}^{n}}{2 \Delta z} \\
\text { Upwind: } \\
\frac{\left(\frac{V_{s}^{2}}{2}\right)_{i+1}^{n}-\left(\frac{V_{s}^{2}}{2}\right)_{i}^{n}}{\Delta z}\end{array}$ & $\begin{array}{l}\text { Central difference: } \\
\frac{\left[V_{s}{ }^{n} V_{s}{ }^{n+1}-\left(\frac{V_{s}}{2}\right)^{n}\right]_{i+1}-\left[V_{s}{ }^{n} V_{s}^{n+1}-\left(\frac{V_{s}^{2}}{2}\right)^{n}\right]_{i-1}}{2 \Delta z} \\
\text { Upwind: } \\
\frac{\left[V_{s}{ }^{n} V_{s}{ }^{n+1}-\left(\frac{V_{s}^{2}}{2}\right)^{n}\right]_{i+1}-\left[V_{s}^{n} V_{s}^{n+1}-\left(\frac{V_{s}^{2}}{2}\right)^{n}\right]_{i}}{\Delta z}\end{array}$ & $B_{2}=\frac{-\rho_{1}(1-\varepsilon)^{2}}{\varepsilon}+\varepsilon \rho_{s}$ \\
\hline \multirow[t]{2}{*}{3} & $V_{s}$ & $\left(V_{s}\right)_{i}^{n}$ & $\left(V_{s}\right)_{i}^{n+1}$ & $C=-\frac{\rho_{1}}{\varepsilon} \frac{\partial \varepsilon}{\partial t}$ \\
\hline & & & & $D=\frac{\rho_{1}\left(\varepsilon_{0} V_{0}\right)}{\varepsilon} \frac{\partial \varepsilon}{\partial t}$ \\
\hline \multirow[t]{4}{*}{4} & & & & $E=\frac{\rho_{1}}{\varepsilon^{2}}\left(\varepsilon_{0} V_{0}\right)^{2} \frac{\partial \varepsilon}{\partial z}$ \\
\hline & $V_{s}$ & $\left(V_{s}\right)_{i}^{n}$ & $\left(V_{s}\right)_{i}^{n+1}$ & $F=\frac{\rho_{1}}{\varepsilon^{2}}\left(\varepsilon_{0} V_{0}\right) \frac{\partial \varepsilon}{\partial z}$ \\
\hline & $V_{s}$ & $\left(V_{s}\right)_{i}^{n}$ & $\left(V_{s}\right)_{i}^{n+1}$ & $G_{1}=\frac{-\rho_{1}}{\varepsilon^{2}} \varepsilon_{0} V_{0}(1-\varepsilon) \frac{\partial \varepsilon}{\partial z}$ \\
\hline & $\left(V_{s}\right)^{2}$ & {$\left[\left(V_{s}\right)^{2}\right]_{i}^{n}$} & $\begin{array}{l}{\left[\left(V_{s}\right)^{2}\right]_{I}^{n+1}} \\
=2\left(V_{s}\right)_{i}^{n}\left(V_{s}\right)_{I}^{n+1}-\left[\left(V_{s}\right)^{2}\right]_{I}^{n}\end{array}$ & $G_{2}=\frac{\rho_{1}}{\varepsilon^{2}}(1-\varepsilon) \frac{\partial \varepsilon}{\partial z}$ \\
\hline 5 & & & & $B=-\mathbf{p}_{2} \frac{\partial\left(\varepsilon_{0} V_{0}\right)}{\partial t}$ \\
\hline 6 & & & & $I=\varepsilon g\left(\rho_{1}-\rho_{s}\right)$ \\
\hline \multirow[t]{2}{*}{7} & & & & $J=\frac{F}{(1-\varepsilon)} \varepsilon_{0} V_{0}$ \\
\hline & $V_{s}$ & $\left\langle V_{s}\right)_{i}^{n}$ & $\left(V_{B}\right)_{1}^{n+1}$ & $K=-\frac{F}{(1-\varepsilon)}$ \\
\hline 8 & & & & $L=\frac{\varepsilon}{(1-\varepsilon) m_{\mathrm{v}}} \frac{\partial \varepsilon}{\partial z}$ \\
\hline
\end{tabular}

reduced to a tridiagonal system and solved with the Thomas algorithm (Anderson et al. 1984).

\section{Central Difference versus Upwind with Implicit Formulation}

The momentum governing equation was discretized with either a central difference or upwind formulation for those terms with the spatial derivative $\partial V_{s} / \partial z$ (the terms with the $B_{1}$ and $B_{2}$ coefficients shown in Table 1). The central difference term adds numerical dispersive error, whereas the upwind formulation adds numerical diffusive error. Model simulations were tried with both formulations and had little effect on the model predictions. The calibrated model used the central difference formulation. 


\section{Linearizing Momentum Equation}

For the implicit scheme, those terms in (15) with either $\left(V_{s}\right)^{2}$ or $V_{s}\left(\partial V_{s} / \partial z\right)$ were linearized at the $n+1$ time step. Those were the second and fourth terms of (15) (as shown, respectively, with the $B_{2}$ and $G_{2}$ coefficients in Table 1).

A general method for linearizing involves defining the nonlinear term at time step $n+1$, as follows (Anderson et al. 1984):

$$
\left(V_{s}^{2} \text { term }\right)^{n+1} \approx\left(V_{s}^{2} \text { term }\right)^{n}+\left.\frac{\partial\left(V_{s}^{2} \text { term }\right)}{\partial V_{s}}\right|^{n}\left(V_{s}^{n+1}-V_{s}^{n}\right)
$$

The term $\left(V_{s}\right) 2 / 2$ from the second term in (15)

$$
B_{2} V_{s} \frac{\partial V_{s}}{\partial z}=B_{2} \frac{\partial\left(\frac{V_{s}^{2}}{2}\right)}{\partial z}
$$

is linearized as follows:

$$
\begin{aligned}
& \left(\frac{V_{s}^{2}}{2}\right)^{n+1} \approx\left(\frac{V_{s}^{2}}{2}\right)^{n}+\left(\frac{\partial\left(\frac{V_{s}^{2}}{2}\right)}{\partial V_{s}}\right)^{n}\left(V_{s}^{n+1}-V_{s}^{n}\right) \Rightarrow\left(\frac{V_{s}^{2}}{2}\right)^{n+1} \\
& \approx\left(\frac{V_{s}^{2}}{2}\right)^{n}+V_{s}^{n}\left(V_{s}^{n+1}-V_{s}^{n}\right)
\end{aligned}
$$

Combining like terms:

$$
\left(\frac{V_{s}^{2}}{2}\right)^{n+1} \approx V_{s}^{n} V_{s}^{n+1}-\left(\frac{V_{s}^{2}}{2}\right)^{n}
$$

Similarly, the portion of the fourth term of (15), $G_{2} V_{s}^{2}$, is also linearized such that

$$
\left(V_{s}^{2}\right)^{n+1} \approx 2\left(V_{s}^{n} V_{s}^{n+1}\right)-\left(V_{s}^{2}\right)^{n}
$$

The linearized terms are included in the finite-difference form of (15), as shown in Table 1.

\section{Final Form of (15) for Tridiagonal Matrix}

The finite-difference equations were formulated as a general explicit-implicit scheme (see Appendix II). This finite-difference scheme weights the $V_{s}$ terms at time steps $n$ and $n+1$ (as shown in Table 1$)$ by $\theta$ and $(1-\theta)$, respectively. When $\theta=0$ the scheme was fully implicit, and when $\theta=1$, the scheme was fully explicit.

The procedure for writing (15) as a tridiagonal matrix, involved (1) rewriting the weighted finite-difference equation with like terms grouped together; (2) reorganizing the equations; (3) incorporating appropriate boundary conditions; and (4) writing the equations in the tridiagonal matrix form. This procedure is shown in Appendix II. The finite-difference form of the solution for (15) is shown for both the central and upwind schemes.

\section{Artificial Diffusion}

An artificial viscosity or diffusive term was introduced to counteract the mathematical effects of dispersive error introduced by the numerical scheme (Richtmyer and Morton 1967). The artificial viscosity term results in smoothing the shock front in the numerical solution. The term was of the form of the diffusive term, i.e., a second derivative using central finite differences, such as

$$
\omega^{\prime} \rho_{s} \frac{\partial^{2} V_{s}}{\partial z^{2}} \approx \omega^{\prime} \rho_{s}\left(\frac{V_{s_{i+1}}-2 V_{s_{i}}+V_{s_{s-1}}}{\Delta z^{2}}\right)=\omega\left(V_{s_{i-1}}-2 V_{s_{i}}+V_{s_{i+1}}\right)
$$

where $\omega^{\prime}=$ artificial viscosity coefficient $\left(\mathrm{L}^{2} / \mathrm{T}\right)$; and $\omega=$ $\omega^{\prime} \rho_{s} / \Delta z^{2}\left(\mathrm{M} / \mathrm{L}^{3}-\mathrm{T}\right)$. Weighting the $V_{s}$ terms by $\theta$ and $(1-\theta)$, respectively, for the general explicit-implicit scheme

$$
\begin{aligned}
& \omega^{\prime} \rho_{s} \frac{\partial^{2} V_{s}}{\partial z^{2}}=\theta \omega\left(V_{s_{i-1}}-2 V_{s_{i}}+V_{s_{i+1}}\right)^{n} \\
& +(1-\theta) \omega\left(V_{s_{i-1}}-2 V_{s_{i}}+V_{s_{i+1}}\right)^{n+1}
\end{aligned}
$$

Adding the artificial viscosity term, defined in (21), to (49)

$$
\begin{aligned}
& \left(x_{2}-(1-\theta) \omega\right)\left(V_{s}\right)_{i-1}^{n+1}+\left(y_{2}+(1-\theta)(2 \omega)\right)\left(V_{s}\right)_{i}^{n+1} \\
& \quad+\left(z_{2}-(1-\theta) \omega\right)\left(V_{s}\right)_{i+1}^{n+1}=\left(x_{1}+\theta \omega\right)\left(V_{s}\right)_{i-1}^{n} \\
& \quad+\left(y_{1}-\theta(2 \omega)\right)\left(V_{s}\right)_{i}^{n}+\left(z_{1}+\theta \omega\right)\left(V_{s}\right)_{i+1}^{n}+x_{0}
\end{aligned}
$$

And, finally, redefining the $x_{1}, y_{1}, z_{1}$, and $x_{2}, y_{2}$, and $z_{2}$ terms used in (47) and (48) [and (56) and (57)]

$$
\begin{gathered}
x_{2}=x_{2}-(1-\theta) \omega ; \quad Y_{2}=y_{2}+(1-\theta)(2 \omega) \\
Z_{2}=z_{2}-(1-\theta) \omega ; \quad X_{1}=x_{1}+\theta \omega \\
Y_{1}=y_{1}-\theta(2 \omega) ; \quad Z_{1}=z_{1}+\theta \omega
\end{gathered}
$$

These redefined terms were substituted into the tridiagonal matrix solution form in (54) (and similarly for the upwind scheme).

\section{Determination of Constitutive Relationships}

Constitutive relationships or slurry stress-strain and permeability-strain properties were required to model gravity sedimentation and thickening.

\section{Coefficient of Volume Compressibility}

Wells (1990) used the following constitutive equation, which fit the experimental data of both gravity sedimentation and cake filtration of kaolin suspensions, to relate the compressibility coefficient to the porosity:

$$
\sigma^{\prime}(\mathrm{kPa})=1.69 \times 10^{9} \exp (-28.9 \varepsilon)
$$

where

$$
m_{v}\left(\mathrm{kPa}^{-1}\right)=2.04 \times 10^{-11} \exp (28.9 \varepsilon)
$$

Fig. 2 shows a graphical presentation of (24).

\section{Intrinsic Permeability}

Experimental data for kaolin clay suspensions collected at CHESS showed that the intrinsic permeability could be represented as an exponential function of the following general form (Dixon et al. 1976):

$$
k=\alpha \exp (\beta \varepsilon)
$$

Wells and Dick (1993) determined the spatial and temporal distribution of permeability within the filter cake, and a bestfit equation for $\varepsilon<0.65$, as shown in Fig. 3, is as follows:

$$
k_{1}\left(\mathrm{~cm}^{2}\right)=2.7 \times 10^{-16} \exp (20 \varepsilon)
$$

For $\varepsilon<0.65$, the scatter appears to be at the limits of the experimental technique. However, in the higher porosity regions where the cake was growing $(\varepsilon \geq 0.65)$, the data scatter appeared to be greater than the limits of the experimental technique, and an equation with a different set of coefficients 
was proposed. Another exponential equation was used in the upper range $(\varepsilon \geq 0.65)$

$$
k_{2}=\alpha_{2} \exp \left(\beta_{2} \varepsilon\right)
$$

The coefficient $\beta_{2}$ was an input parameter to the model, and the coefficient $\alpha_{2}$ was determined by setting (27) equal to (28) at $\varepsilon=0.65$, that is

$$
\begin{gathered}
2.7 \times 10^{-16} \exp (20 \times 0.65)=\alpha_{2} \exp \left(\beta_{2} \times 0.65\right) \\
\alpha_{2}=2.7 \times 10^{-16} \exp \left(\left(20-\beta_{2}\right) 0.65\right)
\end{gathered}
$$

$\beta_{2}$ remained a fitting parameter that was determined by comparison of porosity model predictions to the data.

In the computer algorithm, the permeability was constrained to be a function of the initial porosity if $\varepsilon>\varepsilon_{\text {initial }}$. This occurred in the upper porosity range, and (27) and (28) became

$$
\begin{gathered}
k_{1}\left(\mathrm{~cm}^{2}\right)=2.7 \times 10^{-16} \exp \left(20 \varepsilon_{\text {initial }}\right) \\
k_{2}=\alpha_{2} \exp \left(\beta_{2} \varepsilon_{\text {initial }}\right)
\end{gathered}
$$

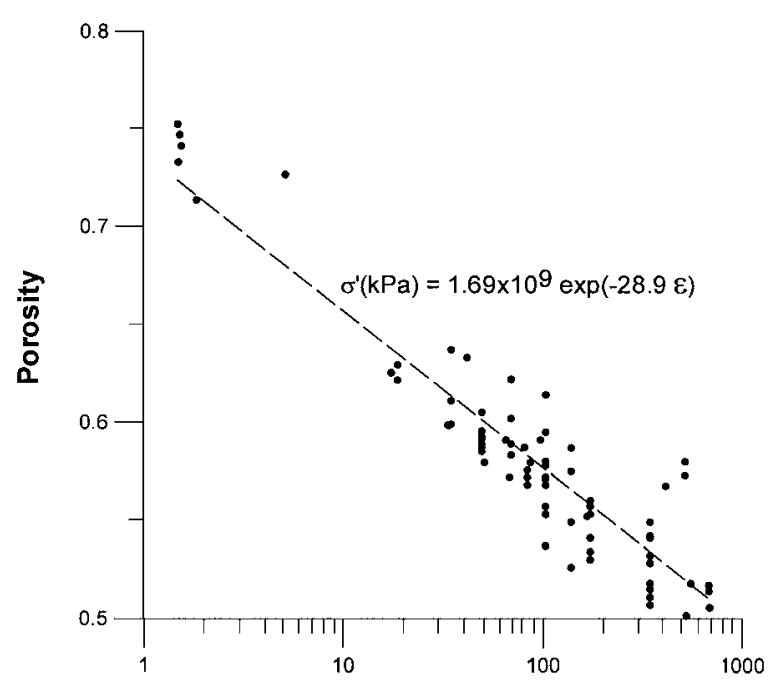

Effective stress, kPa

FIG. 2. Experimental Data and Constitutive Relationship for Porosity $\varepsilon$ versus Effective Stress $\sigma^{\prime}$ (Wells 1990)

\section{MODEL RESULTS}

The model was calibrated by varying the model parameters until model predictions of suspended solids compared well with gravity sedimentation/thickening suspended solids data obtained by Wells (1990) at CHESS. The data were real-time suspended solids concentration measurements at approximately $0.5-\mathrm{mm}$ vertical separation and interpolated to 1 -min intervals. Experimental error in suspended solids concentrations between replicate experiments was on the order of $\pm 8 \%$ (Wells 1990).

The model parameters included $\omega$ (the artificial viscosity), $m_{v}$ (the coefficient of volume compressibility), and the permeability in the higher porosity range. Also, numerical simulations were performed evaluating the effect of the time step $\Delta t$ and difference scheme for the advective term (central difference or upwind) on model predictions.

Wells (1990) obtained four different gravity sedimentation/ thickening data sets for kaolin clay suspensions. The four data sets each had a different initial suspended solids concentration, gravity sedimentation/thickening cell size, temperature, and time period for the experiment. The appropriate initial porosity, cell size, and temperature were input to the model and are summarized in Table 2 . Initial porosity was calculated knowing the initial suspended solids concentration and solids density (assumed to be $2.616 \mathrm{~g} / \mathrm{cm}^{3}$ for kaolin clay), $C_{i}=$ $\rho_{s}(1-\varepsilon)$.

The model was calibrated to Data Set A, which was based on an initial suspended solids concentration of $0.31 \mathrm{~g} / \mathrm{cm}^{3}$, rectangular cell size of $8.1 \mathrm{~cm} \times 1.905 \mathrm{~cm}, 24^{\circ} \mathrm{C}$ temperature, and 16-min duration. The calibrated model used a time step $\Delta t$ of $1 \mathrm{~s}$, the central difference scheme, no artificial diffusion (i.e., $\omega=0$ ), the constitutive relationship for $m_{v}$ of (25) from Wells (1990), and the constitutive relationship for permeability with $\beta_{2}=24$. By substituting $\beta_{2}=24$ into (30), $\alpha_{2}$ was found to be $2.0 \times 10^{-17}$, and (32) became

$$
k_{2}=2.0 \times 10^{-17} \exp (24 \varepsilon)
$$

Fig. 3 is a graphical presentation of $k_{1}[(31)]$ and $k_{2}[(32)]$ superimposed over the permeability data obtained from Wells (1990).

Fig. 4(a) shows the calibrated model predictions of the suspended solids concentration compared with suspended solids Data Set A. Even though the model domain included a pre-

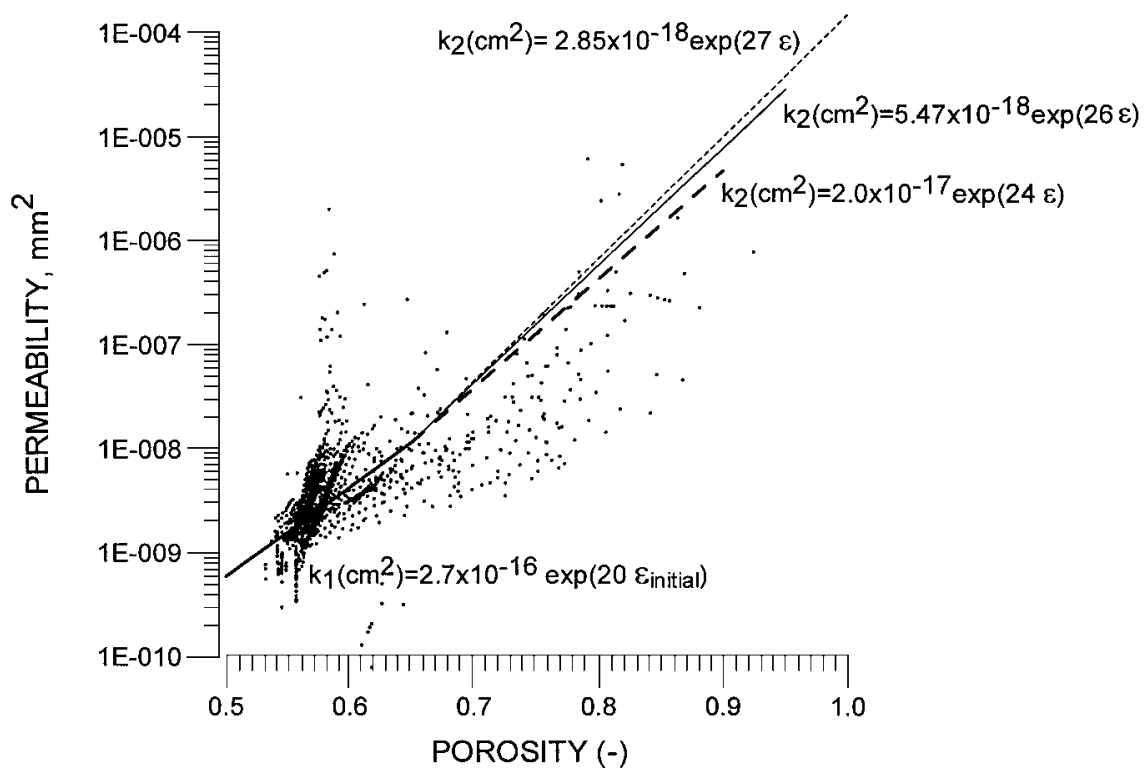

FIG. 3. Comparison of Constitutive Relationships for Permeability $k_{1}[(31)]$ and $k_{2}[(32)]$ and Permeability Data Obtained from Wells (1990) 
TABLE 2. Statistics of Model-Data Comparison for Sedimentation of Kaolin Clay Suspensions

\begin{tabular}{|c|c|c|c|c|c|c|c|c|}
\hline $\begin{array}{l}\text { Data set } \\
\text { (1) }\end{array}$ & $\begin{array}{l}\text { Initial concentration } \\
\left(\mathrm{g} / \mathrm{cm}^{3}\right) \\
(2)\end{array}$ & $\begin{array}{c}\text { Cell size } \\
\left(\mathrm{cm}^{2}\right) \\
(3)\end{array}$ & $\begin{array}{c}\text { Temperature } \\
\left({ }^{\circ} \mathrm{C}\right) \\
(4)\end{array}$ & $\begin{array}{l}\beta_{2} \\
(5)\end{array}$ & $\begin{array}{c}\text { Number of } \\
\text { comparisons } \mathrm{s}^{\mathrm{a}} \\
\text { (6) }\end{array}$ & $\begin{array}{c}\text { Mean error }{ }^{\mathrm{a}} \\
\text { (7) }\end{array}$ & $\begin{array}{l}\text { RMS error }{ }^{a} \\
\text { (8) }\end{array}$ & $\begin{array}{l}\text { Conservation of } \\
\text { mass }^{\mathrm{b}} \\
(\%) \\
(9)\end{array}$ \\
\hline A & 0.31 & 15.4 & 24 & 24 & $\begin{array}{l}233 \\
216\end{array}$ & $\begin{array}{c}0.012 \\
-0.0006\end{array}$ & $\begin{array}{l}0.025 \\
0.0009\end{array}$ & $\begin{array}{l}99.5 \\
99.5\end{array}$ \\
\hline B & 0.48 & 26.3 & 24 & 24 & $\begin{array}{l}379 \\
339\end{array}$ & $\begin{array}{c}0.055 \\
-0.0004\end{array}$ & $\begin{array}{l}0.066 \\
0.001\end{array}$ & $\begin{array}{l}99.5 \\
99.5\end{array}$ \\
\hline & & & & 27 & $\begin{array}{l}702 \\
361\end{array}$ & $\begin{array}{c}0.007 \\
-0.0005\end{array}$ & $\begin{array}{l}0.027 \\
0.001\end{array}$ & $\begin{array}{l}99.7 \\
99.7\end{array}$ \\
\hline $\mathrm{C}$ & 0.15 & 8.0 & 27 & 24 & 181 & 0.041 & 0.096 & 98.5 \\
\hline & & & & & 114 & 0.0124 & 0.0754 & 98.5 \\
\hline & & & & 26 & 196 & 0.005 & 0.050 & 101.2 \\
\hline & & & & & 114 & 0.0085 & 0.074 & 101.2 \\
\hline $\mathrm{D}^{\mathrm{c}}$ & 0.31 & 26.3 & 26 & 24 & 266 & 0.031 & 0.046 & 99.5 \\
\hline & & & & & 239 & -0.0009 & 0.0018 & 99.5 \\
\hline
\end{tabular}

${ }^{\mathrm{a}}$ First number is for suspended solids $\left(\mathrm{g} / \mathrm{cm}^{3}\right)$, and second number is for solid velocity $(\mathrm{mm} / \mathrm{s})$.

${ }^{\mathrm{b}} \mathrm{Based}$ on final mass of solids at the end of simulation divided by initial mass.

${ }^{\mathrm{c}}$ After $20 \mathrm{~min}, \Delta p$ of $15 \mathrm{psi}$ is applied.

a)

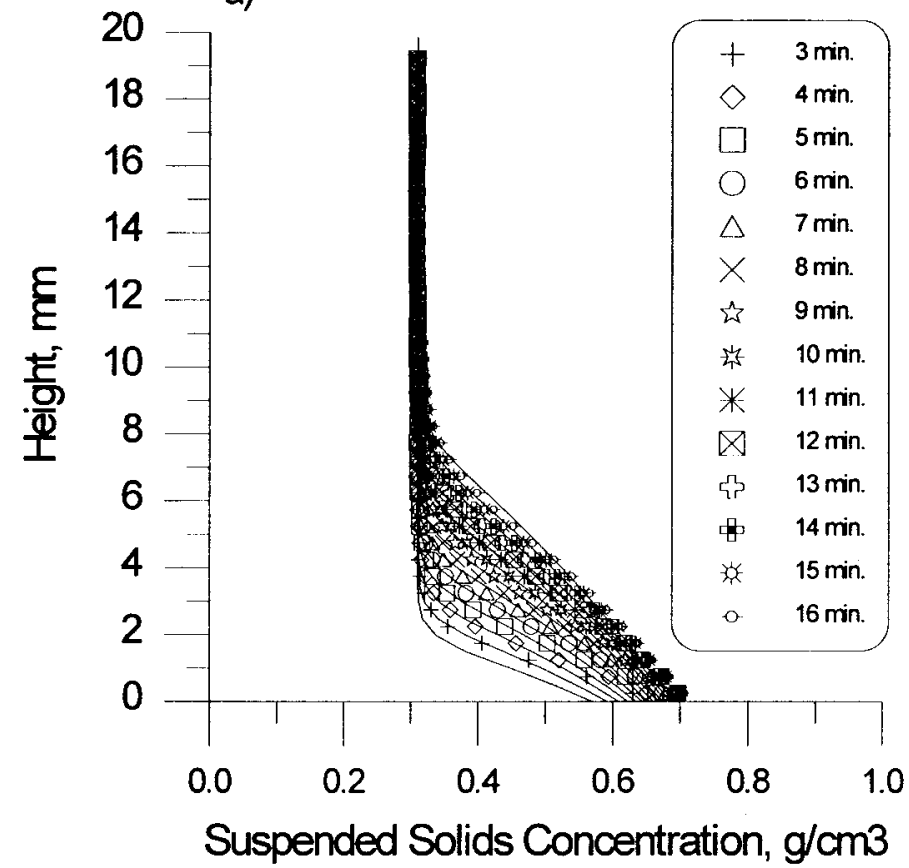

b)

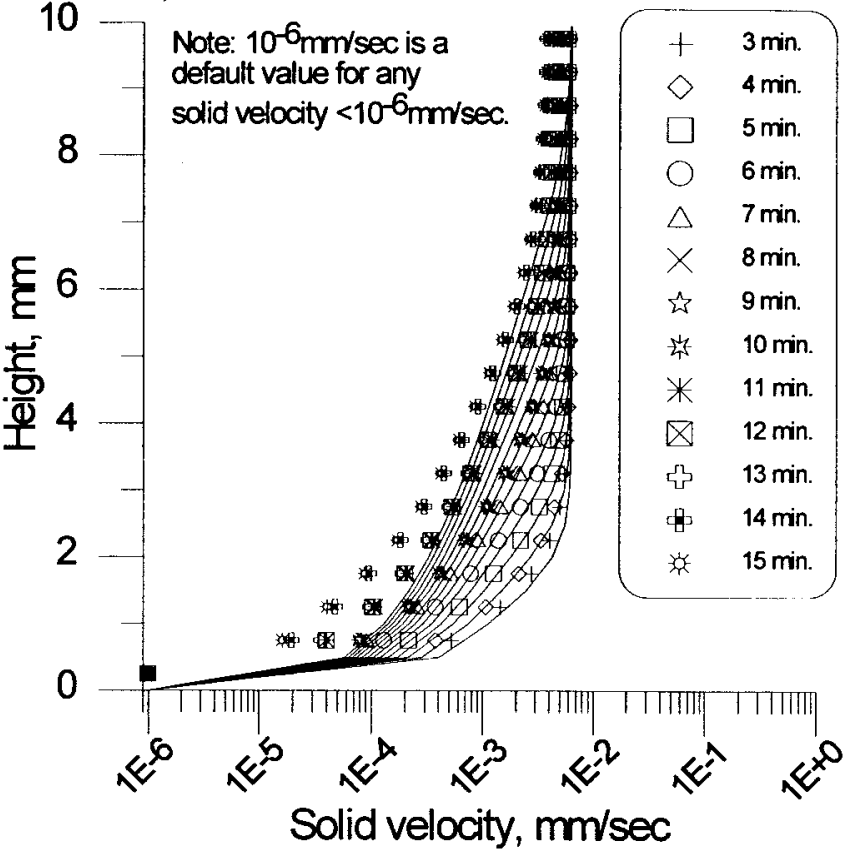

FIG. 4. Comparison of Model Predictions to Data Set A (Medium Initial Concentration)

diction of the clarification at the top of the model domain, CHESS data were limited to the thickening region. The mean error and root-mean-square (RMS) errors between model predictions and data of suspended solids concentration are shown in Table 2.

Solid velocity predictions from the same calibrated model simulation were also compared with solid velocities derived from the data as shown in Fig. 4(b). The solid velocity data were calculated from gravity sedimentation/thickening porosity data as shown in Wells and Dick (1993). The model predictions of solid velocity were calculated from (15). The mean and RMS errors in solid velocities are also shown in Table 2.

\section{Model Verification}

Without changing the model coefficients for the constitutive relationships from those used during the calibration, other simulations were run to verify the validity of the model predictions. As during the calibration, a time step of $1 \mathrm{~s}$ and a central difference scheme were used during the simulations. $\omega$ was zero for the medium and high initial concentrations. In the case of Data Set C, with low initial concentration, the model would not run without artificial viscosity because of high numerical dispersive errors associated with the fast-moving shock front.

Figs. 5-7 show the model results graphically compared with the data for both suspended solids concentrations and solid velocities. Table 2 shows a summary of comparisons between the model and the CHESS data, with their respective mean and RMS errors.

At high initial concentrations, the match of model predictions to experimental data (Data Set B) was not very good for either the suspended solids concentration or solid velocity [Fig. 5(a and b)]. A better fit was obtained by changing the permeability in the higher porosity regions, such that $\beta_{2}=27$. By solving (30), $\alpha_{2}$ was found to be $2.85 \times 10^{-18}$, and therefore

$$
k_{2}=2.85 \times 10^{-18} \exp (27 \varepsilon)
$$

A plot of this value for $k_{2}$ is also included in Fig. 3, and a comparison of the model predictions versus data for $\beta_{2}=27$ is shown in Figs. 5(c and d). 
a) Verification run with $\beta_{2}=24$

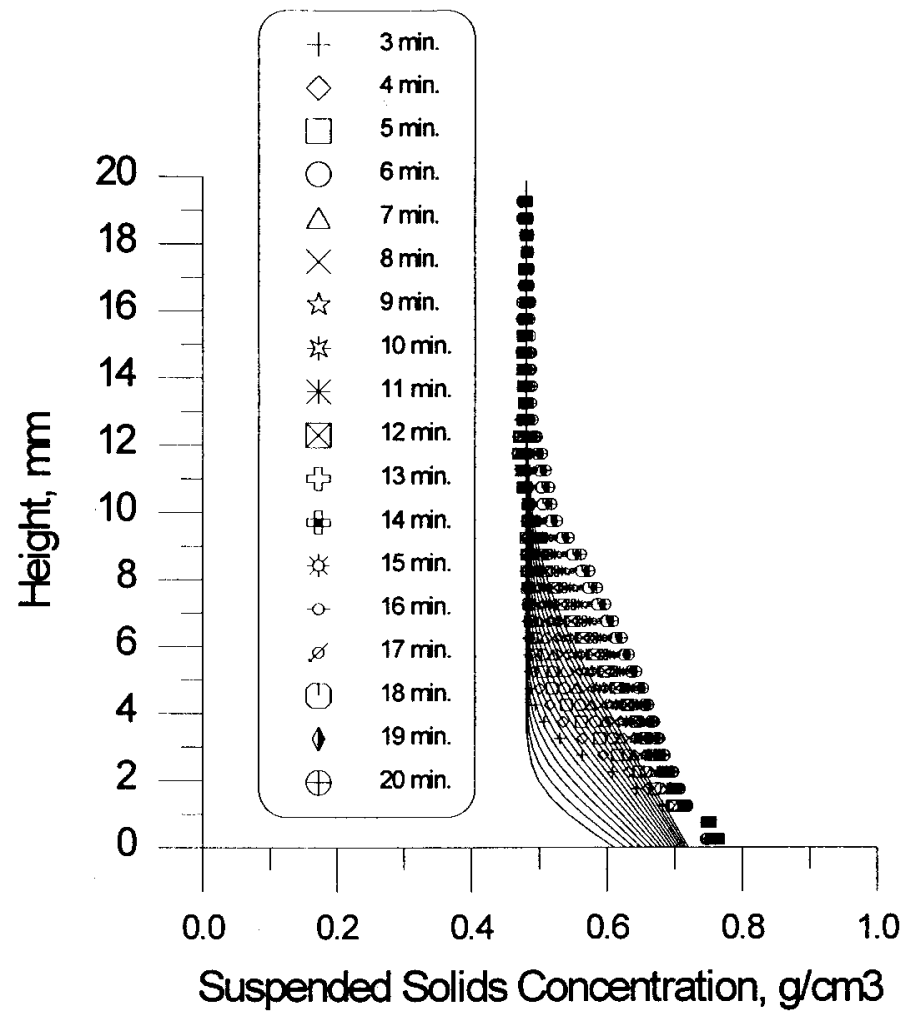

c) Sensitivity run with $\beta_{2}=27$

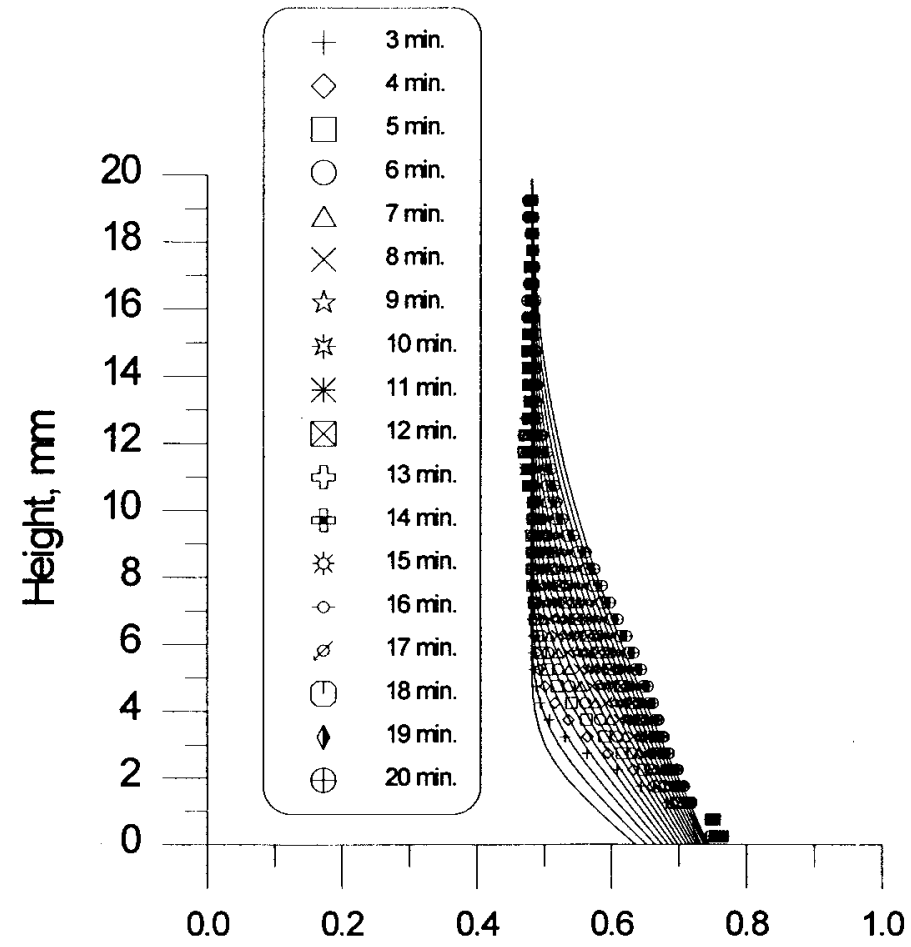

Suspended Solids Concentration, g/cm3
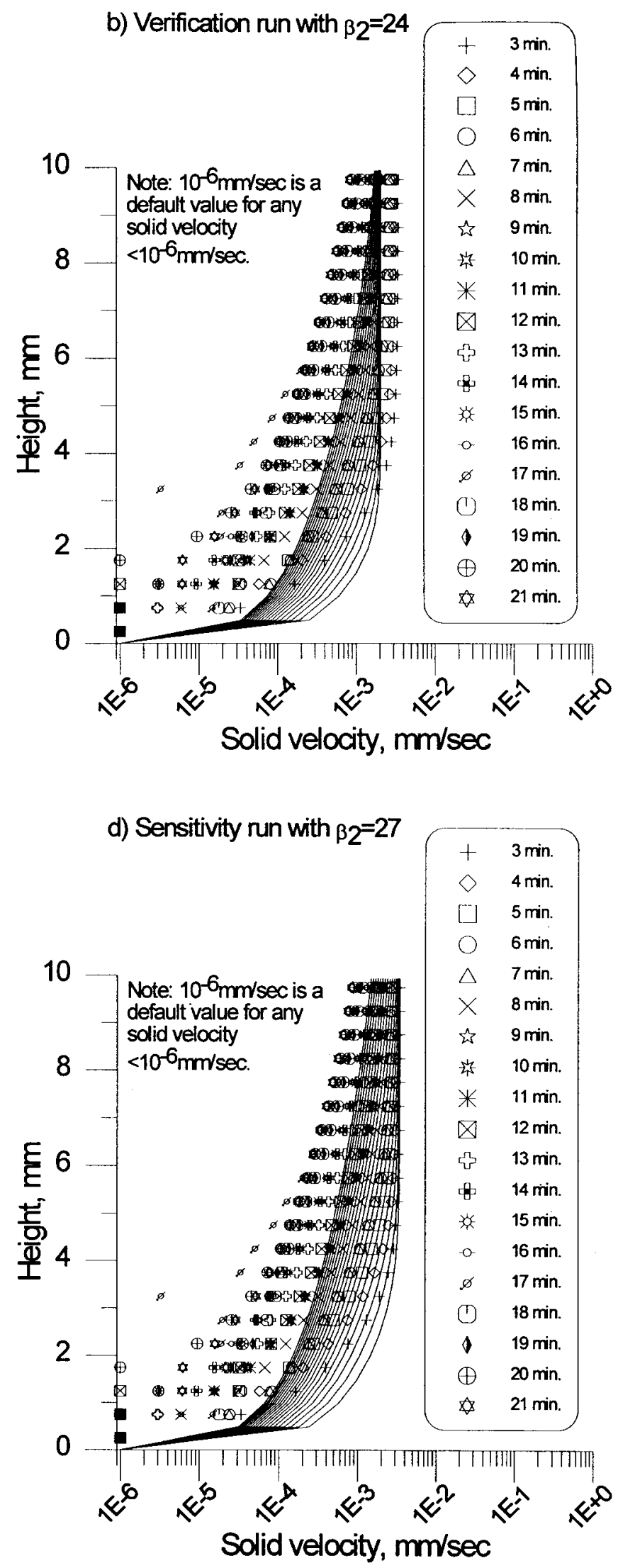

FIG. 5. Comparison of Model Predictions to Data Set B (High Initial Concentration)

Because the model predictions of suspended solids at a low initial concentration (Data Set C) did not match the experimental data well [Figs. 6(a and b)], the constitutive properties (calibrated for the medium concentration) may again not have been adequate. It was found that by setting $\beta_{2}=26$ and solving (30), $\alpha_{2}$ was $5.47 \times 10^{-18}$, and

$$
k_{2}=5.47 \times 10^{-18} \exp (26 \varepsilon)
$$

This permeability relationship is plotted on Fig. 3, and the data-model comparison is shown in Figs. 6(c and d).

Fig. 7 shows that the model-data match of another independent experiment at medium concentration $\left(0.31 \mathrm{~g} / \mathrm{cm}^{3}\right)$ and at 

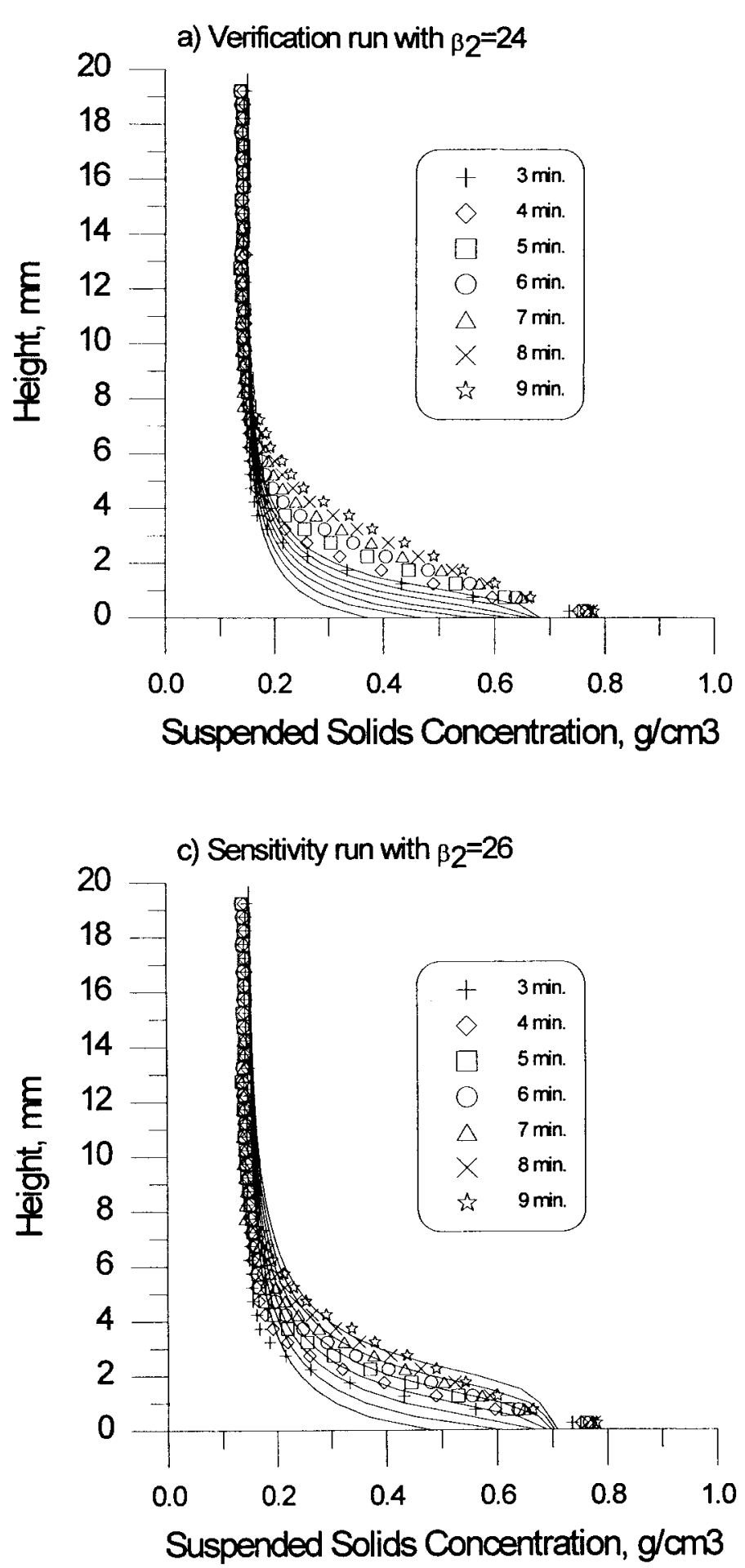
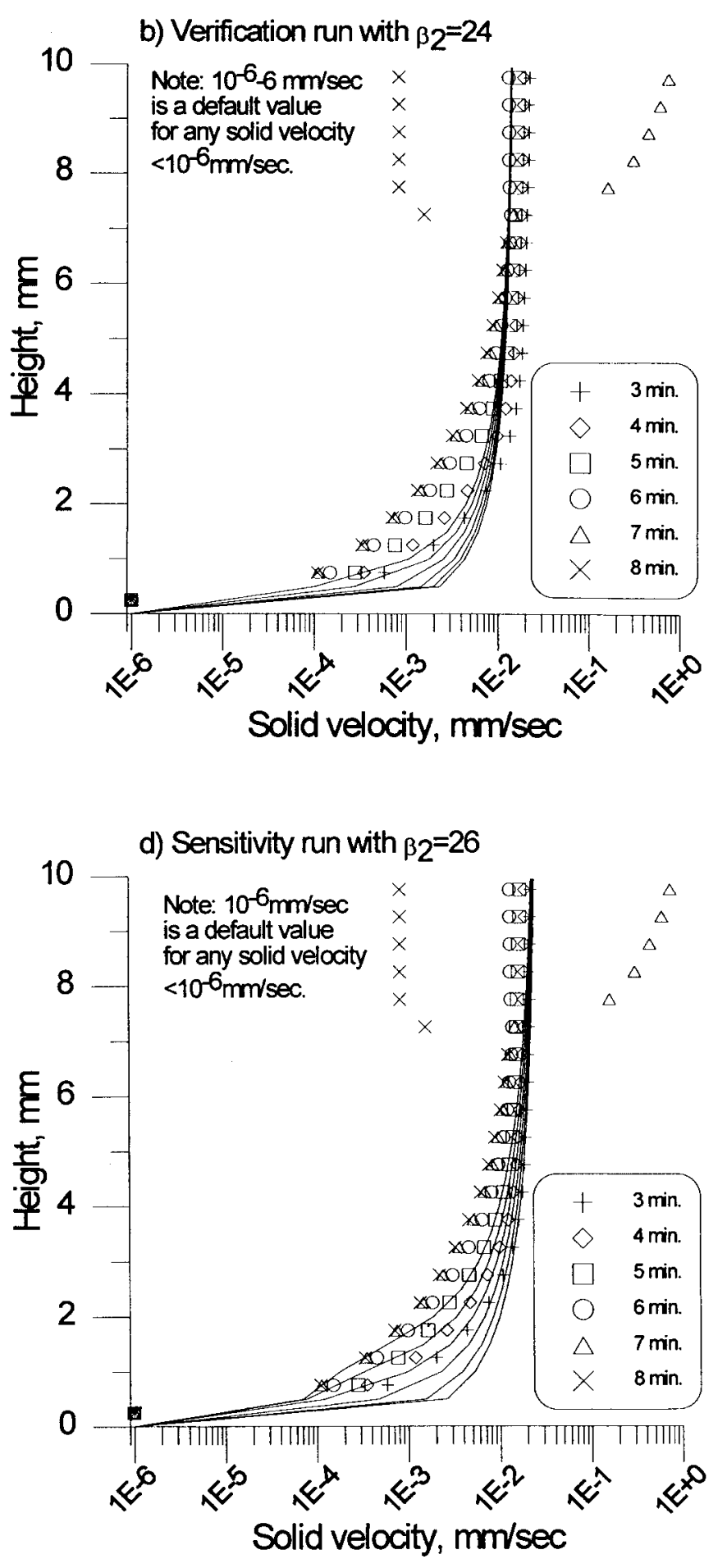

FIG. 6. Comparison of Model Predictions to Data Set C (Low Initial Concentration)

a different temperature (Data Set D) was nearly as close as for the calibrated model. The difference between the data-model match of the calibration (Data Set A) and verification (Data Set D) runs reflects possible variability in the experimental analysis.

\section{Model Sensitivity}

Model sensitivity to the following parameters was considered: permeability $k$, artificial viscosity $\omega$, central difference versus upwind formulations, time step $\Delta t$, and degree of explicitness/implicitness $\theta$. The model was relatively insensitive to the time step, the degree of explicitness/implicitness (with $\theta>0.5)$ or which formulation was used.

\section{Central Difference versus Upwind}

The central difference and upwind formulations were compared for the parameters of Data Set A, with an initial suspended solids concentration of $0.31 \mathrm{~g} / \mathrm{cm}^{3}$. The model predictions were essentially the same for the two formulations, indicating that the convective acceleration term (where the two schemes were applied) was very small. This is shown in a later section, which compares the orders of magnitude of the different terms of the equation.

\section{Permeability}

Suspended solids profiles were compared between the calibrated coefficient value for the upper range $(\varepsilon \geq 0.65), \beta_{2}=$ 
a)

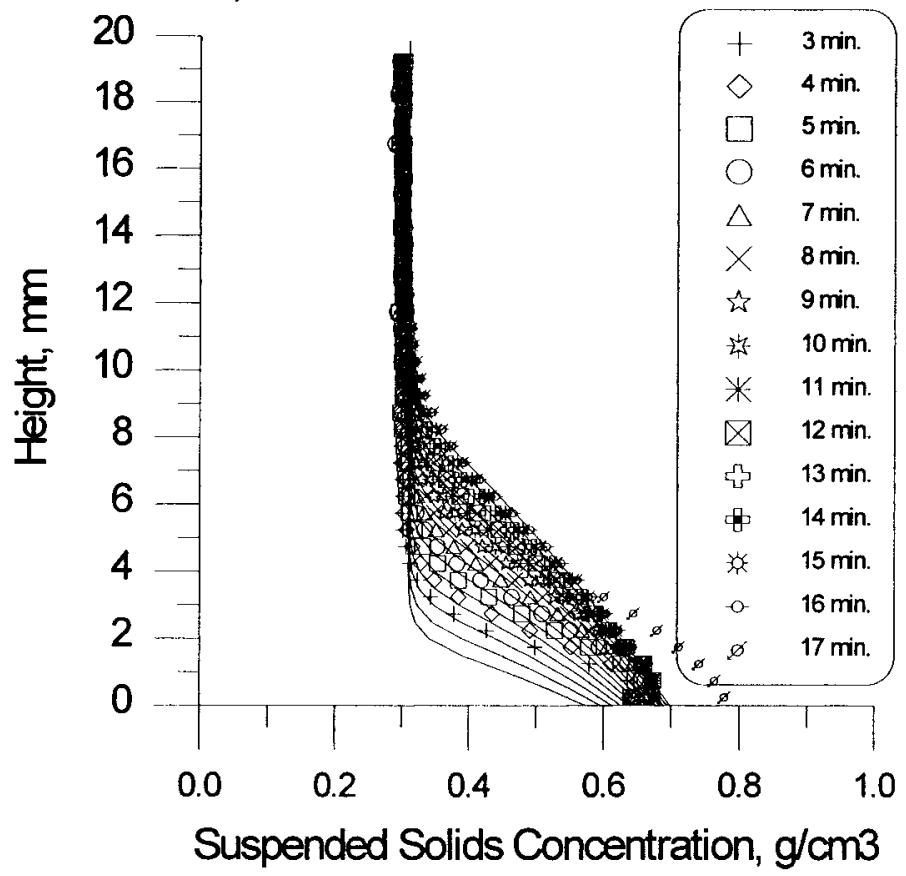

b)

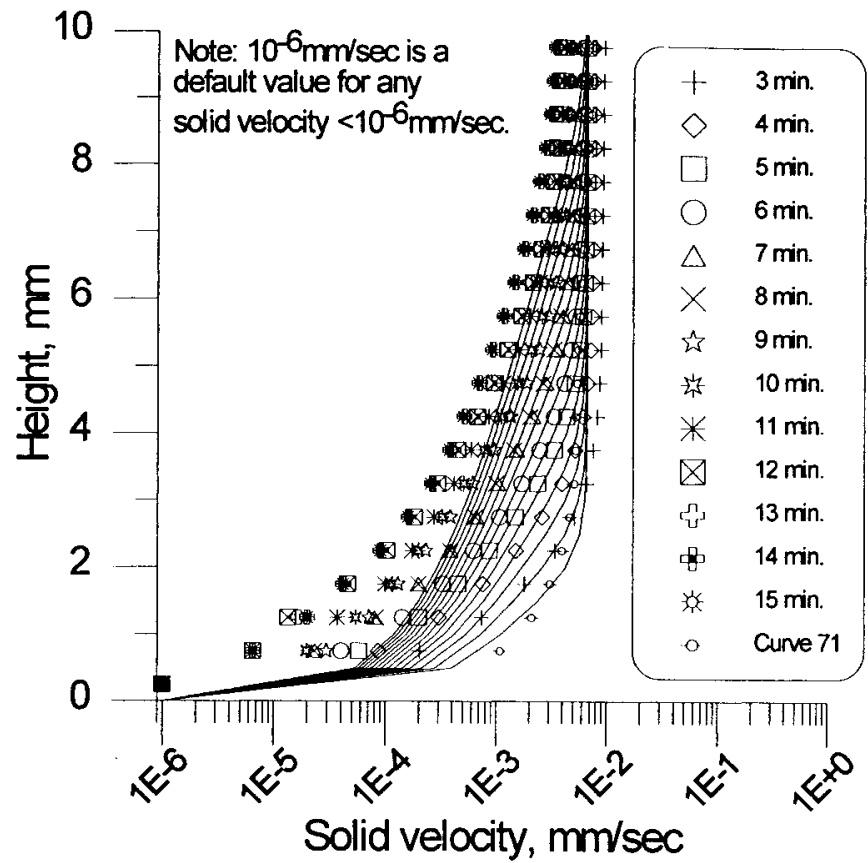

FIG. 7. Comparison of Model Predictions to Data Set D (Medium Initial Concentration)

24 , and values of $\beta_{2}=27$ (for Data Set $B$ ) and $\beta_{2}=26$ (for Data Set $C$ ). This difference had a significant effect, as shown in Figs. 5 and 6. Predictions of suspended solids for the increased $\beta_{2}$ predicted higher concentrations at the bottom.

\section{Artificial Diffusion}

The $\omega$ factor had a significant effect at the bottom of the suspension where the shock front of suspended solids propagated upward. Fig. 8 compares $\omega=2 \times 10^{7} \mathrm{~g} / \mathrm{cm}^{3}-\mathrm{s}$ (used in the verification run of Data Set $\mathrm{C}$ for the low initial concentration with $\beta_{2}=24$ ) to $\omega=2 \times 10^{8} \mathrm{~g} / \mathrm{cm}^{3}$-s. For both the 3and 5-min model predictions of suspended solids (based on $\Delta t$ $=1$ and the central difference scheme for convective acceleration term), the higher $\omega$ resulted in much lower concentrations at the bottom and slightly increased concentrations at the midheights of the suspension because of adding numerical diffusion. At $\Delta t=1 \mathrm{~s}$, the simulations with low initial concentration were unstable unless $\omega$ was greater than approximately $2 \times 10^{6} \mathrm{~g} / \mathrm{cm}^{3}$-s.

\section{Magnitude of Terms}

Fig. 9 shows the relative magnitude of terms given by the model for medium initial concentrations. For all cases (low, medium, and high initial concentrations) the dominant processes were gravity, drag, and, within the thickening cake, effective stress. The initial terms (inertial acceleration and convective acceleration) were many orders of magnitude less than gravity. The lower the initial concentration, however, the larger the initial terms, as shown in Fig. 10.

\section{SUMMARY AND CONCLUSIONS}

A sedimentation and thickening model was developed, calibrated, and verified using the data from the sedimentation/ thickening of kaolin clay suspensions. The gravity sedimentation and thickening model was based on the liquid and solid continuity and liquid and solid momentum equations. The inertial and gravity terms were retained in the gravity sedimentation and thickening model. Numerical simulation demon-

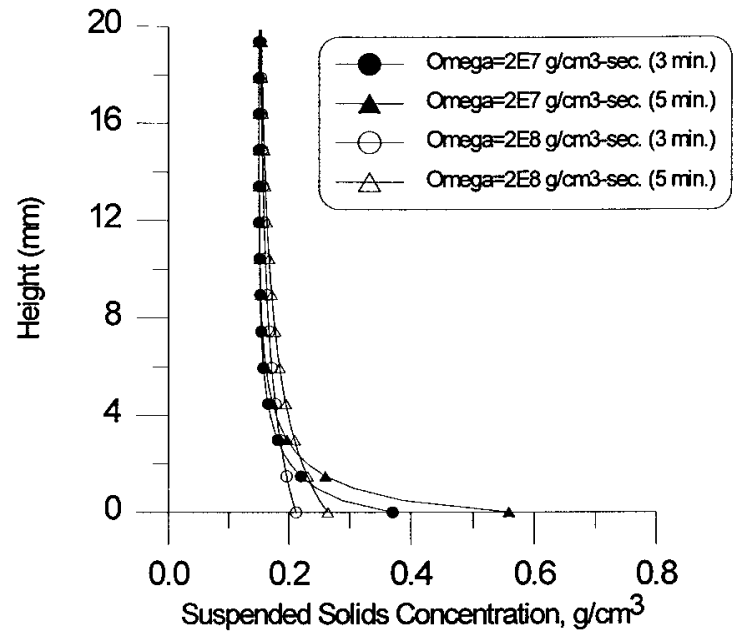

FIG. 8. Sensitivity of Model Predictions to Artificial Viscosity (for Data Set C)

strated that the inertial term was negligible for kaolin suspensions, even at low initial concentrations.

The governing equations were solved by a finite-difference method using a space-staggered mesh. Nonlinear terms in solid velocity were linearized. Boundary conditions and constitutive relationships were determined by evaluation of the data and by model calibration.

The model was extremely sensitive to the constitutive relationships used. Correlations of the calibrated model predictions to data of porosity and solid velocity were good after model calibration. The solid and liquid mass, in general, was conserved during the numerical simulations. Model runs with low initial concentration required the addition of artificial viscosity to remain stable. Improved numerical techniques that reduce numerical diffusion but preclude the use of artificial viscosity for convection-dominated or low initial concentration suspensions could also have been used. For example, the quadratic upwinding technique of Leonard (1979) could have been used.

The permeability relationship determined by model calibration was within the range of the experimental data from Wells 

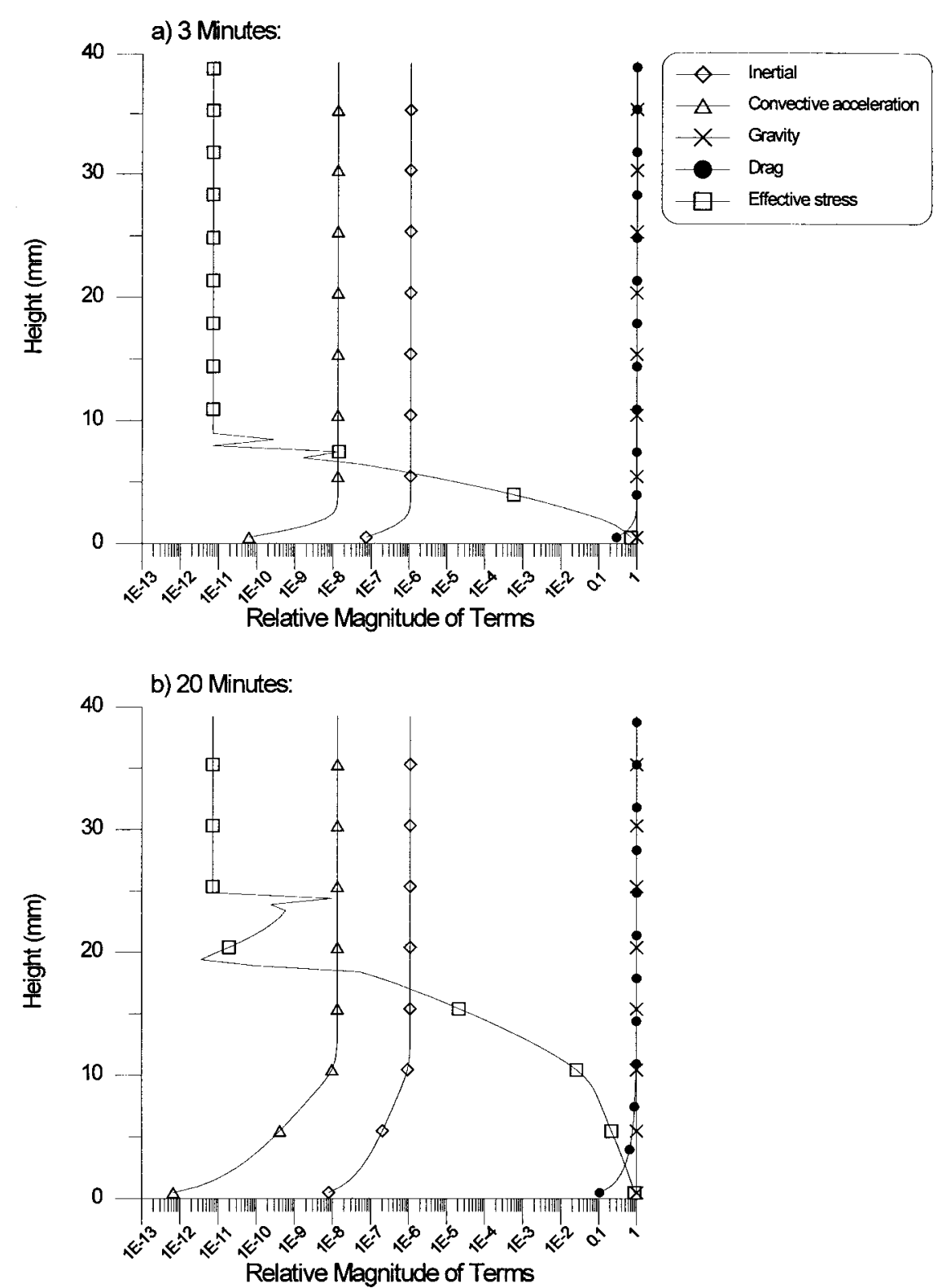

FIG. 9. Relative Magnitude of Terms of (15) Compared to Gravity (Gravity Term Is “1”) for Calibration Run of Data Set A (Medium Initial Concentration)

(1990), even though there was much scatter in the experimental data.

The gravity, drag, and effective stress (within the thickening region) terms were significant, whereas the initial terms were many orders of magnitude less than these terms. However, as the initial concentration of the suspension was reduced, the inertial terms became more important.

As shown by the model's sensitivity to the constitutive relationships, the slurry properties are particularly important for modeling solid-liquid separation processes. Further research is necessary to understand and determine slurry constitutive properties that could then be used in solid-liquid separation models.

This model is useful as a research tool for understanding the effect of the slurry constitutive properties on gravity sedimentation/thickening. Understanding these properties can lead to improvements in modeling solid-liquid separation processes.

\section{APPENDIX I. DERIVATION OF (10) (COMBINED CONTINUITY AND MOMENTUM)}

The technique described below simplifies the four governing equations [(1)-(4)] and the constitutive relationship [(5)] to a single equation for $V_{s}$.
Solving both solid momentum [(4)] and total momentum [(9)] for $-\partial p / \partial z$

Solid momentum:

$$
\begin{aligned}
- & \frac{\partial p}{\partial z}=\rho_{s} \frac{\partial V_{s}}{\partial t}+\rho_{s} V_{s} \frac{\partial V_{s}}{\partial z}+\rho_{s} g-\frac{\varepsilon F}{1-\varepsilon}\left(V_{1}-V_{s}\right) \\
& +\frac{1}{1-\varepsilon} \frac{\partial \sigma^{\prime}}{\partial z}
\end{aligned}
$$

Total momentum:

$$
\begin{aligned}
& -\frac{\partial p}{\partial z}=\rho_{l} \varepsilon \frac{\partial V_{l}}{\partial t}+\rho_{l} \varepsilon V_{l} \frac{\partial V_{l}}{\partial z}+(1-\varepsilon) \rho_{s} \frac{\partial V_{s}}{\partial t} \\
& +(1-\varepsilon) \rho_{s} V_{s} \frac{\partial V_{s}}{\partial z}=+\left[(1-\varepsilon) \rho_{s}+\varepsilon \rho_{l}\right] g+\frac{\partial \sigma^{\prime}}{\partial z}
\end{aligned}
$$

Equating the solid and total momentum equations [(36) and (37)]

$$
\rho_{s} \frac{\partial V_{s}}{\partial t}+\rho_{s} V_{s} \frac{\partial V_{s}}{\partial z}+\rho_{s} g-\frac{\varepsilon F}{1-\varepsilon}\left(V_{l}-V_{s}\right)+\frac{1}{1-\varepsilon} \frac{\partial \sigma^{\prime}}{\partial z}
$$



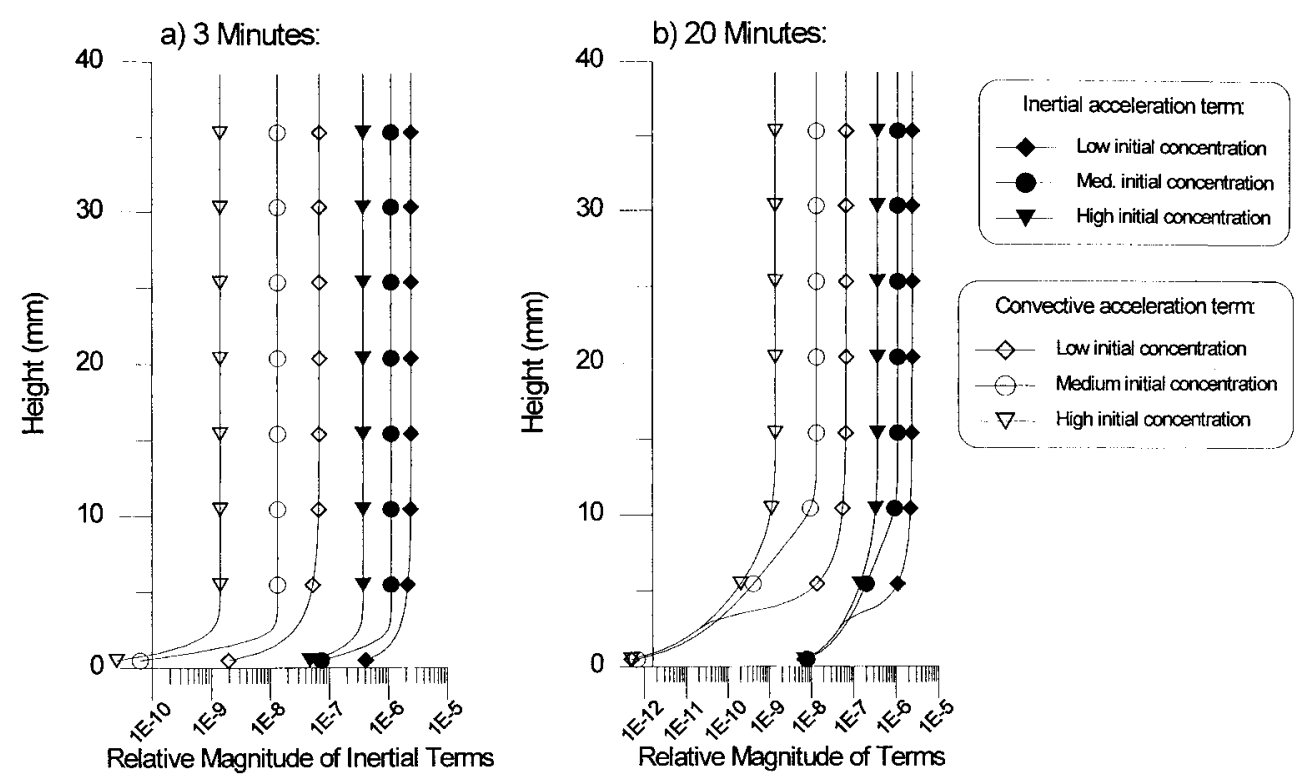

FIG. 10. Comparison of Inertial Terms at Low, Medium, and High Initial Concentrations Relative to Gravity

$$
\begin{aligned}
& =\rho_{l} \varepsilon \frac{\partial V_{l}}{\partial t}+\rho_{l} \varepsilon V_{l} \frac{\partial V_{l}}{\partial z}+(1-\varepsilon) \rho_{s} \frac{\partial V_{s}}{\partial t}+(1-\varepsilon) \rho_{s} V_{s} \frac{\partial V_{s}}{\partial z} \\
& =\left[(1-\varepsilon) \rho_{s}+\varepsilon \rho_{l}\right] g+\frac{\partial \sigma^{\prime}}{\partial z}
\end{aligned}
$$

Substituting the liquid velocity $V_{l}$, from total continuity [(8)], into the first term on the right-hand side of (38)

$$
\begin{aligned}
\rho_{l} \varepsilon \frac{\partial V_{l}}{\partial t} & =\rho_{l} \varepsilon \frac{\partial}{\partial t}\left(\frac{\varepsilon_{0} V_{0}-(1-\varepsilon) V_{s}}{\varepsilon}\right) \\
& =\rho_{l} \varepsilon\left[\varepsilon_{0} V_{0} \frac{\partial\left(\frac{1}{\varepsilon}\right)}{\partial t}+\frac{1}{\varepsilon} \frac{\partial\left(\varepsilon_{0} V_{0}\right)}{\partial t}-\left(\frac{1-\varepsilon}{\varepsilon}\right) \frac{\partial V_{s}}{\partial t}\right. \\
- & \left.V_{s} \frac{\partial\left(\frac{1-\varepsilon}{\varepsilon}\right)}{\partial t}\right]=\rho_{l} \varepsilon\left[\frac{-\varepsilon_{0} V_{0}}{\varepsilon^{2}} \frac{\partial \varepsilon}{\partial t}+\frac{1}{\varepsilon} \frac{\partial\left(\varepsilon_{0} V_{0}\right)}{\partial t}\right. \\
- & \left.\frac{(1-\varepsilon)}{\varepsilon} \frac{\partial V_{s}}{\partial t}+\frac{V_{s}}{\varepsilon^{2}} \frac{\partial \varepsilon}{\partial t}\right]=\rho_{l} \varepsilon\left[\frac{-(1-\varepsilon)}{\varepsilon} \frac{\partial V_{s}}{\partial t}\right. \\
+ & \left.\frac{\left(V_{s}-\varepsilon_{0} V_{0}\right)}{\varepsilon^{2}} \frac{\partial \varepsilon}{\partial t}+\frac{1}{\varepsilon} \frac{\partial\left(\varepsilon_{0} V_{0}\right)}{\partial t}\right]
\end{aligned}
$$

Substituting the liquid velocity $V_{l}$, from total continuity [(8)], into the second term on the right-hand side of (38)

$$
\begin{aligned}
& \rho_{l} \varepsilon V_{l} \frac{\partial V_{l}}{\partial z}=\rho_{l} \varepsilon\left[\frac{\varepsilon_{0} V_{0}-(1-\varepsilon) V_{s}}{\varepsilon}\right] \frac{\partial}{\partial z}\left[\frac{\varepsilon_{0} V_{0}-(1-\varepsilon) V_{s}}{\varepsilon}\right] \\
& =\rho_{l}\left[\varepsilon_{0} V_{0}-(1-\varepsilon) V_{s}\right]\left[\frac{1}{\varepsilon} \frac{\partial\left(\varepsilon_{0} V_{0}\right)}{\partial z}+\varepsilon_{0} V_{0} \frac{\partial\left(\frac{1}{\varepsilon}\right)}{\partial z}\right. \\
& \left.-V_{s} \frac{\partial(1-\varepsilon)}{\partial z}-\frac{(1-\varepsilon)}{\varepsilon} \frac{\partial V_{s}}{\partial z}\right] \\
& =\rho_{l}\left[\varepsilon_{0} V_{0}-(1-\varepsilon) V_{s}\right]\left[\frac{-\varepsilon_{0} V_{0}}{\varepsilon^{2}} \frac{\partial \varepsilon}{\partial z}+\frac{V_{s}}{\varepsilon^{2}} \frac{\partial \varepsilon}{\partial z}-\frac{(1-\varepsilon)}{\varepsilon} \frac{\partial V_{s}}{\partial z}\right]
\end{aligned}
$$

$$
=\rho_{l}\left[\varepsilon_{0} V_{0}-(1-\varepsilon) V_{s}\right]\left[\frac{V_{s}-\varepsilon_{0} V_{0}}{\varepsilon^{2}} \frac{\partial \varepsilon}{\partial z}-\frac{(1-\varepsilon)}{\varepsilon} \frac{\partial V_{s}}{\partial z}\right]
$$

Substituting (39) and (40) into (38)

$$
\begin{aligned}
& \rho_{s} \frac{\partial V_{s}}{\partial t}+\rho_{s} V_{s} \frac{\partial V_{s}}{\partial z}+\rho_{s} g-\frac{\varepsilon F}{1-\varepsilon}\left(V_{l}-V_{s}\right)+\frac{1}{1-\varepsilon} \frac{\partial \sigma^{\prime}}{\partial z} \\
& \quad=\rho_{l} \varepsilon\left[\frac{-(1-\varepsilon)}{\varepsilon} \frac{\partial V_{s}}{\partial t}+\frac{\left(V_{s}-\varepsilon_{0} V_{0}\right)}{\varepsilon^{2}} \frac{\partial \varepsilon}{\partial t}+\frac{1}{\varepsilon} \frac{\partial\left(\varepsilon_{0} V_{0}\right)}{\partial t}\right] \\
& \quad+\rho_{l}\left[\varepsilon_{0} V_{0}-(1-\varepsilon) V_{s}\right]\left[\frac{V_{s}-\varepsilon_{0} V_{0}}{\varepsilon^{2}} \frac{\partial \varepsilon}{\partial z}-\frac{(1-\varepsilon)}{\varepsilon} \frac{\partial V_{s}}{\partial z}\right] \\
& \quad+(1-\varepsilon) \rho_{s} \frac{\partial V_{s}}{\partial t}+(1-\varepsilon) \rho_{s} V_{s} \frac{\partial V_{s}}{\partial z} \\
& \quad=+\left[(1-\varepsilon) \rho_{s}+\varepsilon \rho_{l}\right] g+\frac{\partial \sigma^{\prime}}{\partial z}
\end{aligned}
$$

Organizing and simplifying terms

$$
\begin{aligned}
& {\left[(1-\varepsilon) \rho_{l}+\varepsilon \rho_{s}\right] \frac{\partial V_{s}}{\partial t}} \\
& +\left[\rho_{l}\left[\varepsilon_{0} V_{0}-(1-\varepsilon) V_{s}\right]\left(\frac{1-\varepsilon}{\varepsilon}\right)+\varepsilon \rho_{s} V_{s}\right] \frac{\partial V_{s}}{\partial z} \\
& +\left[-\rho_{l} \frac{\left(V_{s}-\varepsilon_{0} V_{0}\right.}{\varepsilon}\right] \frac{\partial \varepsilon}{\partial t} \\
& +\left[\frac{\rho_{l}}{\varepsilon^{2}}\left(\varepsilon_{0} V_{0}-V_{s}\right)\left(\varepsilon_{0} V_{0}-(1-\varepsilon) V_{s}\right)\right] \frac{\partial \varepsilon}{\partial z}-\rho_{l} \frac{\partial\left(\varepsilon_{0} V_{0}\right)}{\partial t} \\
& =\varepsilon g\left(\rho_{l}-\rho_{s}\right)+\frac{\varepsilon F}{(1-\varepsilon)}\left(\frac{\varepsilon_{0} V_{0}-(1-\varepsilon) V_{s}-\varepsilon V_{s}}{\varepsilon}\right) \\
& +\left[-\frac{1}{(1-\varepsilon)}+1\right] \frac{\partial \sigma^{\prime}}{\partial z}
\end{aligned}
$$

Substituting the constitutive relationship [(5)] into (42)

$$
\begin{aligned}
& {\left[(1-\varepsilon) \rho_{l}+\varepsilon \rho_{s}\right] \frac{\partial V_{s}}{\partial t}} \\
& \quad+\left[\rho_{l}\left[\varepsilon_{0} V_{0}-(1-\varepsilon) V_{s}\right]\left(\frac{1-\varepsilon}{\varepsilon}\right)+\varepsilon \rho_{s} V_{s}\right] \frac{\partial V_{s}}{\partial z}
\end{aligned}
$$




$$
\begin{aligned}
& +\left[-\rho_{l} \frac{\left(V_{s}-\varepsilon_{0} V_{0}\right.}{\varepsilon}\right] \frac{\partial \varepsilon}{\partial t} \\
& +\left[\frac{\rho_{l}}{\varepsilon^{2}}\left(\varepsilon_{0} V_{0}-V_{s}\right)\left(\varepsilon_{0} V_{0}-(1-\varepsilon) V_{s}\right)\right] \frac{\partial \varepsilon}{\partial z} \\
& -\rho_{l} \frac{\partial\left(\varepsilon_{0} V_{0}\right)}{\partial t}=\varepsilon g\left(\rho_{l}-\rho_{s}\right)+\left(\frac{F}{(1-\varepsilon)}\right)\left(\varepsilon_{0} V_{0}-V_{s}\right) \\
& +\frac{\varepsilon}{(1-\varepsilon) m_{v}} \frac{\partial \varepsilon}{\partial z}
\end{aligned}
$$

After grouping terms, (10) is obtained in the main text.

\section{APPENDIX II. DEVELOPMENT OF FINITE- DIFFERENCE FORM OF (15)}

\section{Central Difference Scheme}

A weighted average of the finite-difference approximation in (15) is determined by weighting the $V_{s}$ terms at time steps $n$ and $n+1$, by $\theta$ and $(1-\theta)$

$$
\begin{aligned}
A & {\left[\frac{\left(V_{s}\right)_{i}^{n+1}-\left(V_{s}\right)_{i}^{n}}{\Delta t}\right] } \\
& +B_{1}\left[\theta\left(\frac{\left(V_{s}\right)_{i+1}^{n}-\left(V_{s}\right)_{i-1}^{n}}{2 \Delta z}\right)+(1-\theta)\left(\frac{\left(V_{s}\right)_{i+1}^{n+1}-\left(V_{s}\right)_{l-1}^{n+1}}{2 \Delta z}\right)\right] \\
& +B_{2}\left[\theta\left(\frac{\left(\frac{V_{s}^{2}}{2}\right)_{i+1}^{n}-\left(\frac{V_{s}^{2}}{2}\right)_{i-1}^{n}}{2 \Delta z}\right)\right. \\
& \left.+(1-\theta)\left(\frac{\left.V_{s}^{n} V_{s}^{n+1}-\left(\frac{V_{s}^{2}}{2}\right)^{n}\right]-\left[V_{s}^{n} V_{s}^{n+1}-\left(\frac{V_{s}^{2}}{2}\right)^{n}\right]}{2 \Delta z}\right)\right] \\
& +C\left[\theta\left(V_{s}\right)_{i}^{n}+(1-\theta)\left(V_{s}\right)_{i}^{n+1}\right]+D+E \\
& +F\left[\theta\left(V_{s}\right)_{i}^{n}+(1-\theta)\left(V_{s}\right)_{i}^{n+1}\right]+G_{1}\left[\theta\left(V_{s}\right)_{i}^{n}+(1-\theta)\left(V_{s}\right)_{i}^{n+1}\right] \\
& +G_{2}\left[\theta\left(V_{s}^{2}\right)_{i}^{n}+(1-\theta)\left(2\left(V_{s}\right)_{i}^{n}\left(V_{s}\right)_{i}^{n+1}-\left(V_{s}^{2}\right)_{i}^{n}\right)\right]+H \\
& \left.=I+J+K\left[\theta V_{s}\right)_{i}^{n}+(1-\theta)\left(V_{s}\right)_{i}^{n+1}\right]+L
\end{aligned}
$$

Rewriting and grouping like terms

$$
\begin{aligned}
& {\left[\frac{-(1-\theta)\left(B_{1}+B_{2}\left(V_{s}\right)_{i-1}^{n}\right)}{2 \Delta z}\right]\left(V_{s}\right)_{i-1}^{n+1}} \\
& \quad+\left[\frac{A}{\Delta t}+(1-\theta)\left(C+F+G_{1}+2 G_{2}\left(V_{s}\right)_{i}^{n}-K\right)\right]\left(V_{s}\right)_{i}^{n+1} \\
& \quad+\left[\frac{(1-\theta)\left(B_{1}+B_{2}\left(V_{s}\right)_{i+1}^{n}\right)}{2 \Delta z}\right]\left(V_{s}\right)_{i+1}^{n+1} \\
& \quad=\left[\frac{\theta B_{1}}{2 \Delta z}-\frac{(1-2 \theta) B_{2}\left(V_{s}\right)_{i-1}^{n}}{4 \Delta z}\right]\left(V_{s}\right)_{i-1}^{n} \\
& \quad+\left[\frac{A}{\Delta t}+\theta\left(-C-F-G_{1}+K\right)+(1-2 \theta) G_{2}\left(V_{s}\right)_{i}^{n}\right]\left(V_{s}\right)_{i}^{n} \\
& \quad+\left[-\frac{\theta B_{1}}{2 \Delta z}-\frac{(1-2 \theta) B_{2}\left(V_{s}\right)_{i+1}^{n}}{4 \Delta z}\right]\left(V_{s}\right)_{i+1}^{n} \\
& \quad+(-D-E-H+I+J+L)
\end{aligned}
$$

The terms can be redefined as follows, to set up the tridiagonal system:

$$
X_{0}=-D-E-H+I+J+L
$$

Explicit terms:

$$
\begin{gathered}
X_{1}=\frac{\theta B_{1}}{2 \Delta z}-\frac{(1-2 \theta) B_{2}\left(V_{s}\right)_{i-1}^{n}}{4 \Delta z} \\
Y_{1}=\frac{A}{\Delta t}+\theta\left(-C-F-G_{1}+K\right)+(1-2 \theta) G_{2}\left(V_{s}\right)_{i}^{n} \\
Z_{1}=\frac{-\theta B_{1}}{2 \Delta z}+\frac{(1-2 \theta) B_{2}\left(V_{s}\right)_{i+1}^{n}}{4 \Delta z}
\end{gathered}
$$

Implicit terms:

$$
\begin{gathered}
X_{2}=\frac{-(1-\theta)\left(B_{1}+B_{2}\left(V_{s}\right)_{i-1}^{n}\right)}{2 \Delta z} \\
Y_{2}=\frac{A}{\Delta t}+(1-\theta)\left(C+F+G_{1}+2 G_{2}\left(V_{s}\right)_{i}^{n}-K\right) \\
Z_{2}=\frac{(1-\theta)}{2 \Delta z}\left(B_{1}+B_{2}\left(V_{s}\right)_{i+1}^{n}\right)
\end{gathered}
$$

Substituting the terms for $x_{0}, x_{1}, y_{1}, z_{1}, x_{2}, y_{2}$, and $z_{2}$ into (45):

$$
\begin{aligned}
& x_{2}\left(V_{s}\right)_{i-1}^{n+1}+y_{2}\left(V_{s}\right)_{i}^{n+1}+z_{2}\left(V_{s}\right)_{i+1}^{n+1} \\
& \quad=x_{1}\left(V_{s}\right)_{i-1}^{n}+y_{1}\left(V_{s}\right)_{i}^{n}+z_{1}\left(V_{s}\right)_{i+1}^{n}+x_{0}
\end{aligned}
$$

Boundary conditions:

$$
\text { Bottom: } \quad\left(V_{s}\right)_{1}=0
$$

$$
\text { Top: } \frac{\partial V_{s}}{\partial z}=0 \quad \text { or } \quad\left(V_{s}\right)_{k+2}^{n}=\left(V_{s}\right)_{k}^{n}, \quad\left(V_{s}\right)_{k+2}^{n+1}=\left(V_{s}\right)_{k}^{n+1}
$$

Substituting $i=k+1$ into (49):

$$
\begin{aligned}
& x_{2}\left(V_{s}\right)_{k}^{n+1}+y_{2}\left(V_{s}\right)_{k+1}^{n+1}+z_{2}\left(V_{s}\right)_{k+2}^{n+1} \\
& \quad=x_{1}\left(V_{s}\right)_{k}^{n}+y_{1}\left(V_{s}\right)_{k+1}^{n}+z_{1}\left(V_{s}\right)_{k+2}^{n}+x_{0}
\end{aligned}
$$

Substituting the top boundary condition $[(51)]$ into (52):

$$
\left(x_{2}+z_{2}\right)\left(V_{s}\right)_{k}^{n+1}+y_{2}\left(V_{s}\right)_{k+1}^{n+1}=\left(x_{1}+z_{1}\right)\left(V_{s}\right)_{k}^{n}+y_{1}\left(V_{s}\right)_{k+1}^{n}+x_{0}
$$

The following is the matrix representing the system of equations to be solved at each time step for all $\left(V_{s}\right)^{n+1}$ (the righthand side of the equations represent the knowns):

$\left[\begin{array}{ccccccccc}1 & & & & & & & & \\ 0 & x_{2} & y_{2} & z_{2} & & & & & \\ 0 & 0 & x_{2} & y_{2} & z_{2} & & & & \\ \vdots & & & \ddots & \ddots & \ddots & & & \\ \vdots & & & & & \ddots & \ddots & \ddots & \\ 0 & 0 & 0 & 0 & 0 & 0 & x_{2} & y_{2} & z_{2} \\ 0 & 0 & 0 & 0 & 0 & 0 & 0 & x_{2}+z_{2} & y_{2}\end{array}\right]\left[\begin{array}{c}\left(V_{s}\right)_{1}^{n+1} \\ \left(V_{s}\right)_{2}^{n+1} \\ \left(V_{s}\right)_{3}^{n+1} \\ \vdots \\ \left(V_{S}\right)_{K}^{n+1} \\ \left(V_{s}\right)_{K+1}^{n+1}\end{array}\right]$

$$
=\left[\begin{array}{cccc}
0 & & & \\
x_{1}\left(V_{s}\right)_{1} & +y_{1}\left(V_{s}\right)_{2} & z_{1}\left(V_{s}\right)_{3} & +x_{0} \\
x_{1}\left(V_{s}\right)_{2} & y_{1}\left(V_{s}\right)_{3} & z_{1}\left(V_{s}\right)_{4} & +x_{0} \\
\vdots & & & \\
x_{1}\left(V_{s}\right)_{K-1} & +y_{1}\left(V_{s}\right)_{K} & +z_{1}\left(V_{s}\right)_{K+1} & +x_{0} \\
+\left(x_{1}+z_{1}\right)\left(V_{s}\right)_{K} & +y_{1} V_{s_{K+1}} & +0 & +x_{0}
\end{array}\right]
$$

\section{Upwind Scheme}

A similar development included an upwind scheme for the advective term. Again, the weighted average of the finite-difference approximation is determined, as follows: 


$$
\begin{aligned}
A & {\left[\frac{\left(V_{s}\right)_{i}^{n+1}-\left(V_{s}\right)_{i}^{n}}{\Delta t}\right]+B_{1}\left[\theta\left(\frac{\left(V_{s}\right)_{i+1}^{n}-\left(V_{s}\right)_{i}^{n}}{\Delta z}\right)\right.} \\
& \left.+(1-\theta)\left(\frac{\left(V_{s}\right)_{i+1}^{n+1}-\left(V_{s}\right)_{i}^{n+1}}{\Delta z}\right)\right]+B_{2}\left[\theta\left(\frac{\left(\frac{V_{s}^{2}}{2}\right)_{i+1}^{n}-\left(\frac{V_{s}^{2}}{2}\right)_{i}^{n}}{\Delta z}\right)^{n}\right. \\
& \left.+(1-\theta)\left(\frac{\left.\left.\left[V_{s}^{n} V_{s}^{n+1}-\left(\frac{V_{s}^{2}}{2}\right)^{n}\right]-\left[V_{s}^{n} V_{s}^{n+1}-\left(\frac{V_{s}^{2}}{2}\right)^{n}\right]\right)\right]}{\Delta z}\right)\right] \\
& +C\left[\theta\left(V_{s}\right)_{i}^{n}+(1-\theta)\left(V_{s}\right)_{i}^{n+1}\right]+D+E \\
& +F\left[\theta\left(V_{s}\right)_{i}^{n}+(1-\theta)\left(V_{s}\right)_{i}^{n+1}\right]+G_{1}\left[\theta\left(V_{s}\right)_{i}^{n}+(1-\theta)\left(V_{s}\right)_{i}^{n+1}\right] \\
& +G_{2}\left[\theta\left(V_{s}^{2}\right)_{i}^{n}+(1-\theta)\left(2\left(V_{s}\right)_{i}^{n}\left(V_{s}^{n}\right)_{i}^{n+1}-\left(V_{s}^{2}\right)_{i}^{n}\right)\right]+H \\
& =I+J+K\left[\theta\left(V_{s}\right)_{i}^{n}+(1-\theta)\left(V_{s}\right)_{i}^{n+1}\right]+L
\end{aligned}
$$

Following the same procedures as used for the central difference scheme, the solution matrix of (54) is also valid for the upwind scheme except for the definitions of the following parameters:

Explicit terms:

$$
\begin{gathered}
X_{1}=0 \\
Y_{1}=\frac{A}{\Delta t}+\theta\left(\frac{B_{1}}{\Delta z}-C-F-G_{1}+K\right) \\
+(1-2 \theta)\left(\frac{-B_{2}}{2 \Delta z}+G_{2}\right)\left(V_{s}\right)_{i}^{n} \\
Z_{1}=\frac{-\theta B_{1}}{\Delta z}+\frac{(1-2 \theta) B_{2}}{2 \Delta z}\left(V_{s}\right)_{i+1}^{n}
\end{gathered}
$$

Implicit terms:

$$
X_{2}=0
$$

$$
\begin{aligned}
& Y_{2}=\frac{A}{\Delta t}+(1-\theta) \\
& \cdot\left[\left(\frac{-B_{1}-B_{2}\left(V_{s}\right)_{i}^{n}}{\Delta z}\right)+C+F+G_{1}+2 G_{2}\left(V_{s}\right)_{i}^{n}-K\right] \\
& Z_{2}=\frac{(1-\theta)\left(B_{1}+B_{2}\left(V_{s}\right)_{i+1}^{n}\right)}{\Delta z}
\end{aligned}
$$

\section{ACKNOWLEDGMENTS}

The writers acknowledge the support of the National Science Foundation Directorate of Engineering grants CEE-8414614 and CES8821199 .

\section{APPENDIX III. REFERENCES}

Adams, E., and Rodi, W. (1990). "Modeling flow and mixing of sedimentation tanks."' J. Hydr. Engrg., ASCE, 116, 895-913.

Anderson, D. A., Tannehill, J. C., and Pletcher, R. H. (1984). Computational fluid mechanics and heat transfer. Hemisphere Publishing Corp., Washington, D.C.

Been, K. (1980). "Stress strain behaviour of a cohesive soil deposited under water," $\mathrm{PhD}$ dissertation, University of Oxford, Oxford, U.K.

Bierck, B. R., Wells, S. A., and Dick, R. I. (1988). "Compressible cake filtration: Monitoring cake formation and shrinkage using synchrotron X-rays."' J. Water Pollution Control Fed., 60(5), 645-649.

Coe, H. S., and Clevenger, G. H. (1916). "Methods for determining the capacities of slime-settling tanks." Trans. Am. Inst. of Mining, Metallurgical and Petr. Engrs., 55, 356.

Concha, F., and Bustos, M. C. (1985). "Theory of sedimentation of flocculated fine particles."' Proc., Engrg. Found. Conf., Flocculation, Sed- imentation and Consolidation, B. M. Moudgil and P. Somasundaran, eds., $39-55$.

Das, B. (1983). Advanced soil mechanics. McGraw-Hill, New York.

Dixon, D. C. (1979). "Theory of gravity thickening." Progress in filtration and separation, R. J. Wakeman, ed., Elsevier Science, Amsterdam.

Dixon, D. C., Souter, P., and Buchanan, J. E. (1976). Chemical Engrg. Sci., 31, 737.

Dixon, D. C., Souter, P., and Buchanan, J. E. (1985). “Argument not invalidated.' Filtration and Separation, 22, 183.

Evans, B., and Filman, D. (1988). "Solids handling costs at large sewage treatment plants." Proc., ASCE-CSCE Nat. Conf. on Envir. Engrg., 590-595.

Farlow, S. J. (1982). Partial differential equations for scientists and engineers. Wiley, New York.

Fitch, B. (1966). "Current theory and thickener design.' Industrial and Engrg. Chem., 58, 18.

Fitch, B. (1975). "Current theory and thickener design." Filtration and Separation, 12, 355, 480, 636.

Fitch, B. (1979). "Sedimentation of flocculent suspensions: State of the art." Am. Inst. of Chemical Engrg. J., 25(6), 913-930.

Fitch, B. (1983). "Kynch theory and compression zones." Am. Inst. of Chemical Engrg. J., 29, 940-947.

Karl, J. R. (1993). "Gravity sedimentation: A one-dimensional numerical model," MS thesis, Portland State University, Portland, Ore.

Kos, P. (1977). "Gravity thickening of water-treatment-plant sludges." $J$. AWWA, 69(5), 272-282.

Kos, P. (1985). "Sedimentation and thickening-General overview." Proc., Engrg. Found. Conf., Flocculation, Sedimentation and Consolidation, B. M. Moudgil and P. Somasundaran, eds., 39-55.

Kynch, G. J. (1952). “A theory of sedimentation." Trans. Farraday Soc., 48, 166-176.

Leonard, B. P. (1979). "A stable and accurate convective modelling procedure based on quadratic upstream interpolation." Comp. Methods in Appl. Mech. and Engrg., 19, 59-98.

Michaels, A. S., and Bolger, J. C. (1962). "Settling rates and sediment volumes of flocculated kaolin suspensions." Industrial and Engrg. Chem., 1(1), 24-33.

Richardson, J. F., and Zaki, W. N. (1954). "Sedimentation and fluidisation: Part I." Trans. Instn. Chemical Engrs.

Richtmyer, R. D., and Morton, K. W. (1967). Difference methods for initial-value problems. Wiley Interscience Publishers, New York.

Robinson, C. S. (1926). Industrial and Engrg. Chem., 18, 689.

Schiffman, R. L., Pane, V., and Sunara, V. (1985). "Sedimentation and consolidation." Proc., Engrg. Found. Conf., Flocculation, Sedimentation and Consolidation, B. M. Moudgil and P. Somasundaran, eds., $57-121$.

Shirato, M., Kato, H., Kobavashi, K., and Sakazaki, H. (1970). “Analyses of settling of thick slurries due to consolidation." J. Chem. Engrg., Japan, 3, 98.

Soo, S. L. (1989). Particulates and continuum multiphase fluid dynamics. Hemisphere Publishing Corp., New York.

Tiller, F. M. (1981). "Revision of Kynch sedimentation theory." Am. Inst. of Chemical Engrg. J., 27, 823-829.

Vaccari, D. A., and Uchrin, C. G. (1989). "Modeling and simulation of compressive gravity thickening of activated sludge.' J. Envir. Sci. and Health, A24(6), 645-674.

Wakeman, R. J., and Holdich, R. G. (1984). "Theoretical and experimental modeling of solids and liquid pressures in batch sedimentation.' Filtration and Separation, 21, 420-422.

Wells, S. A. (1990). "Compressible cake filtration modeling and analysis." PhD dissertation, Cornell University, Ithaca, N.Y.

Wells, S. A., and Dick, R. I. (1993). "Permeability, liquid and solid velocity, and effective stress variations in compressible cake filtration." Advances in filtration and separation, W. Leung, ed., Vol. 7, American Filtration and Separation Society, 9-12.

Willis, M. S. (1983). "A multiphase theory of filtration." Progress in filtration and separation, R. J. Wakeman, ed., Elsevier Science, New York, 1-56.

\section{APPENDIX IV. NOTATION}

The following symbols are used in this paper:

$c=$ concentration $\left(\mathrm{M} / \mathrm{L}^{3}\right)$;

$e=$ void ratio;

$F=\varepsilon \mu / k$, averaged interfacial interaction term between solid and liquid phases $\left(\mathrm{M} / \mathrm{L}^{3}-\mathrm{T}\right)$;

$g=$ acceleration due to gravity $\left(\mathrm{L} / \mathrm{T}^{2}\right)$;

$k=$ coefficient of permeability, intrinsic permeability $\left(\mathrm{L}^{2}\right)$; 
$m_{v}=$ coefficient of volume compressibility $\left(\mathrm{T}^{2}-\mathrm{L} / \mathrm{M}\right)$;

$n=$ time level;

$p=$ fluid static pressure $\left(\mathrm{M} / \mathrm{L}-\mathrm{T}^{2}\right)$;

$P=$ applied pressure $\left(\mathrm{M} / \mathrm{L}-\mathrm{T}^{2}\right)$;

$P_{l}=$ local pressure of liquid $\left(\mathrm{M} / \mathrm{L}-\mathrm{T}^{2}\right)$;

$P_{s}=$ local pressure of solid $\left(\mathrm{M} / \mathrm{L}-\mathrm{T}^{2}\right)$;

$t=$ time $(\mathrm{T})$;

$V_{l}=$ true liquid velocity (in contrast to Darcy velocity) $(\mathrm{L} / \mathrm{T})$;

$V_{s}=$ velocity of solid particles $(\mathrm{L} / \mathrm{T})$;

$V_{0}=$ true liquid velocity at $z=0(\mathrm{~L} / \mathrm{T})$

$z=$ distance from filtration medium (L);

$\alpha=$ empirical constant $\left(\mathrm{L}^{2}\right)$;

$\varepsilon=$ porosity (volume liquid/total volume); $\varepsilon_{l}=$ empirical constant corresponding to limiting porosity;

$\varepsilon_{0}=$ terminal porosity at $z=0$;

$\zeta=$ empirical constant $\left(\mathrm{M} / \mathrm{L}-\mathrm{T}^{2}\right)$

$\theta=$ explicitness/implicitness $(\theta=0$ fully implicit; $\theta=1$ fully explicit);

$\mu=$ dynamic (or absolute) viscosity $(\mathrm{M} / \mathrm{L}-\mathrm{T})$;

$\rho_{l}=$ liquid density $\left(\mathrm{M} / \mathrm{L}^{3}\right)$;

$\rho_{s}=$ solid density $\left(\mathrm{M} / \mathrm{L}^{3}\right)$;

$\rho_{w}=$ density of water (weight per unit of its own volume)(M/ $\mathrm{L}^{3}$ );

$\sigma=$ total stress applied to system (M/L-T $\left.{ }^{2}\right) ;$

$\sigma^{\prime}=$ effective stress (interparticle pressure) $\left(\mathrm{M} / \mathrm{L}^{-\mathrm{T}^{2}}\right)$;

$\omega=$ artificial viscosity $\left(\mathrm{M} / \mathrm{L}^{3}-\mathrm{T}\right)$; and

$\omega^{\prime}=$ artificial viscosity coefficient $\left(\mathrm{L}^{2} / \mathrm{T}\right)$. 